\title{
Ab initio coupled-cluster approach to nuclear structure with modern nucleon-nucleon interactions
}

\author{
G. Hagen, ${ }^{1}$ T. Papenbrock, ${ }^{2,1}$ D. J. Dean, ${ }^{1}$ and M. Hjorth-Jensen ${ }^{3}$ \\ ${ }^{1}$ Physics Division, Oak Ridge National Laboratory, Oak Ridge, TN 37831, USA \\ ${ }^{2}$ Department of Physics and Astronomy, University of Tennessee, Knoxville, TN 37996, USA \\ ${ }^{3}$ Department of Physics and Center of Mathematics for Applications, University of Oslo, N-0316 Oslo, Norway
}

\begin{abstract}
We perform coupled-cluster calculations for the doubly magic nuclei ${ }^{4} \mathrm{He},{ }^{16} \mathrm{O},{ }^{40,48} \mathrm{Ca}$, for neutronrich isotopes of oxygen and fluorine, and employ "bare" and secondary renormalized nucleon-nucleon interactions. For the nucleon-nucleon interaction from chiral effective field theory at order nextto-next-to-next-to leading order, we find that the coupled-cluster approximation including triples corrections binds nuclei within $0.4 \mathrm{MeV}$ per nucleon compared to data. We employ interactions from a resolution-scale dependent similarity renormalization group transformations and assess the validity of power counting estimates in medium-mass nuclei. We find that the missing contributions due to three-nucleon forces are consistent with these estimates. For the unitary correlator model potential, we find a slow convergence with respect to increasing the size of the model space. For the $G$-matrix approach, we find a weak dependence of ground-state energies on the starting energy combined with a rather slow convergence with respect to increasing model spaces. We also analyze the center-of-mass problem and present a practical and efficient solution.
\end{abstract}

PACS numbers: 21.10.Dr, 21.60.-n, 31.15.Dv, 21.30.-x

\section{INTRODUCTION}

In the last decade, ab-initio nuclear structure calculations have made great progress [1-9]. Light nuclei up to carbon or so can now be described in terms of their nucleonic degrees of freedom, realistic nucleon-nucleon (NN) forces (i.e., those that fit the available body of $\mathrm{NN}$ data with a $\chi^{2} \approx 1$ per datum) augmented by a threenucleon force (3NF). One of the major advances is due to the systematic construction of nuclear forces within chiral effective field theory (EFT) 10 15]. Within this approach, the unknown short-range part of the nuclear force is systematically encoded in terms of low-energy constants and contact terms, while the long-range part of the interaction stems from pion exchange. One of the hallmarks of this approach is the "power counting", i.e., an expansion of the nuclear Lagrangian in terms of the momentum ratio $Q / \Lambda_{\chi}$. Here, $Q$ denotes the typical momentum scale at which the nucleus is probed, while $\Lambda_{\chi}$ denotes the high-momentum cutoff scale that limits the applicability of the effective field theory. Within this approach, three-nucleon forces appear naturally at next-tonext-to-leading $\left(\mathrm{N}^{2} \mathrm{LO}\right)$ order $\left(Q / \Lambda_{\chi}\right)^{3}$, and four-nucleon forces get introduced at $\mathrm{N}^{3} \mathrm{LO}$, that is at order $\left(Q / \Lambda_{\chi}\right)^{4}$ in terms of the momentum scale and the cutoff $\Lambda_{\chi}$.

The chiral interactions have been tested in very light systems of mass $A=3,4$ through precise few-body calculations [ 3,44 , and in $p$-shell nuclei within no-core shell model (NCSM) calculations [6, 7]. In these calculations, the $\mathrm{NN}$ interaction was taken up to $\mathrm{N}^{3} \mathrm{LO}$, while the three-nucleon interaction was taken at its leading order $\left(Q / \Lambda_{\chi}\right)^{3}\left(\mathrm{~N}^{2} \mathrm{LO}\right)$. Within the NCSM calculations, the "bare" chiral interaction needs to be renormalized, due to the size of the model space. Lattice calculations provide a different approach for the implementation and solution of chiral interactions in the nuclear many-body problem [5]. Presently, such calculations employ twoand three-nucleon forces at order $\mathrm{N}^{2} \mathrm{LO}$, and they target $p$-shell nuclei. The coupled-cluster method is an alternative approach that is particularly suited to study the saturation properties of chiral nucleon-nucleon interactions in medium-mass nuclei such as ${ }^{40,48} \mathrm{Ca}$ and ${ }^{48} \mathrm{Ni}$ [8], or even heavier nuclei. Using a Gamow basis expansion [16], this method can also include continuum effects and describe rare isotopes that are in the vicinity of nuclei with closed (sub)shells [17, 18].

The "bare" chiral interaction models are still relatively "hard" interactions, that is the interaction has nonzero matrix elements even for high momentum transfers $Q \gg k_{F}$, where $k_{F} \approx 1.35 \mathrm{fm}^{-1}$ denotes the Fermi momentum at nuclear saturation. Using for example matrix elements of the chiral interaction by Machleidt and Entem [13], we extend typically the momentum integrations up to $8 \mathrm{fm}^{-1}$. For a model space consisting of oscillator wave functions, a simple estimate shows that the number of oscillator shells required for the solution of a nucleus with radius $R$ and an interaction with momentum cutoff $\lambda$ is about $N \approx \lambda R$ (see Sect. IV] below for details). Recall that the number of single-particle states (single-particle $j$-shells) grows as $N^{3}$ in the $m$-scheme (and as $N^{2}$ in a $j$-coupled spherical scheme). It is therefore clear that high-momentum interactions require large model spaces. This makes wave-function-based solutions of the nuclear many-body problem a challenging task. Within the coupled-cluster approach, one can overcome this difficulty through a spherically symmetric reformulation of the method. This approach enables us to consider much increased model spaces, including even up to 20 oscillator shells. The obvious alternative consists of lowering the momentum cutoff.

The momentum cutoff of the interactions can be decreased by a similarity renormalization group (SRG) 
transformation of the NN interaction [19], or by integrating out high-momentum modes within a renormalization group transformation or a similarity transformation [20, 21]. Such transformations preserve all properties (such as phase shifts and bound states) of the NN interactions up to the cutoff. The SRG transformations yield interactions that are band diagonal in momentum space, and the band width is characterized by a momentum scale. Eigenstates of these interactions are localized in momentum space, and the low-momentum states are thus decoupled from the high-momentum physics. The low-momentum interactions $V_{\text {low } k}$ are characterized by a momentum cutoff beyond which all interaction matrix elements quickly approach zero. We denote the momentum scale of the SRG interactions and the cutoff of the $V_{\text {low } k}$ interactions by $\lambda$. Starting with $\lambda=\Lambda_{\chi}$, one can thus generate a one-parameter family of interactions from the chiral NN interaction models by performing SRG transformations, or by integrating out high-momentum modes. We will use this approach in order to study the scale- or cutoff dependence of the nuclear binding energy, and to examine aspects of the power counting. Throughout this work we make the underlying assumption that a complete description within chiral effective field theory would yield an accurate description of atomic nuclei. Thus, we attribute any scale dependence directly to the neglected three-nucleon forces and other neglected high-order terms of the interaction.

The more traditional way of dealing with "hard" interactions consists of the computation of the so-called $G$-matrix 22], which is based on a Green's function approach [23] using normally unperturbed propagators. The $G$-matrix depends thus on the starting energy that is employed in its construction. Analytical arguments demand that this dependence weakens as increasingly larger model spaces are considered, and it vanishes for infinite spaces. Within the spherical coupled-cluster approach, we are able to investigate the convergence properties of the $G$-matrix that is constructed from chiral interaction models.

Another approach to renormalize "hard" interactions is the unitary correlator method (UCOM). Here a unitary transformation is constructed to remove the hard core and short-range contributions of the tensor force by an appropriately formulated correlation operator [24]. This method has seen several applications, but its convergence and saturation properties have not yet been studied in a framework that includes substantial wave function correlations. We fill this gap in this work.

It is the purpose of this paper to employ "bare" and renormalized nucleon-nucleon interactions (employing SRG and low-momentum techniques, the $G$-matrix and the UCOM method), to compare and analyze their convergence and saturation properties and their impact on nuclear structure. In addition to this task, this paper also contains significant supplemental information that could not be presented in several recent short communications [8, 18, 25 27]. In particular, we present a detailed study and practical solution of the center-of-mass problem, and study the evolution of single-particle energies in neutron-rich oxygen and fluorine isotopes.

This paper is organized as follows. In Section II] we introduce spherical coupled-cluster theory. We address and resolve questions regarding the center-of-mass problem in Section III. In Section IV] we compute the binding energies of the nuclei ${ }^{4} \mathrm{He},{ }^{16} \mathrm{O},{ }^{40} \mathrm{Ca}$, and ${ }^{48} \mathrm{Ca}$ starting from "bare" chiral NN interactions. Section $\mathrm{V}$ is dedicated to the evolution of single particle energies in neutronrich oxygen isotopes. In Section VI, we study ${ }^{40} \mathrm{Ca}$ for several momentum scales $\lambda$ of the SRG interaction, and examine the power counting. Section VII focuses on the starting-energy dependence and the convergence properties of the $G$-matrix for ${ }^{4} \mathrm{He}$ and ${ }^{16} \mathrm{O}$. We analyze the convergence properties of the UCOM interaction in Section VIII We finish with our Summary. Some technical details of the spherical coupled-cluster method are relegated to the Appendix.

\section{SPHERICAL COUPLED-CLUSTER THEORY}

In this section we give an outline of the coupledcluster method [28 39] and introduce an angular momentum coupled formulation of the coupled-cluster equations. First we outline coupled-cluster theory for the computation of ground state energy of closed-shell nuclei within the so-called CCSD and $\Lambda$-CCSD $(\mathrm{T})$ approaches. Thereafter, we introduce the equation-of-motion theory for the calculation of ground and excited states in closedand open-shell nuclei. We introduce also the spherical formulation of coupled-cluster theory. Finally, we discuss how to calculate expectation values of observables in coupled-cluster theory.

\section{A. Coupled-cluster theory for closed-shell nuclei}

Coupled-cluster theory is based on the similarity transformation

$$
\bar{H}=e^{-T} H e^{T}
$$

of the normal-ordered Hamiltonian $H$. Here, the Hamiltonian is normal-ordered with respect to a product state $\left|\phi_{0}\right\rangle$ which serves as a reference. Likewise, the particlehole cluster operator

$$
T=T_{1}+T_{2}+\ldots+T_{A}
$$

is defined with respect to the reference state. The $k$ particle $k$-hole $(k \mathrm{p}-k \mathrm{~h})$ cluster operator is

$$
T_{k}=\frac{1}{(k !)^{2}} \sum_{i_{1}, \ldots, i_{k} ; a_{1}, \ldots, a_{k}} t_{i_{1} \ldots i_{k}}^{a_{1} \ldots a_{k}} a_{a_{1}}^{\dagger} \ldots a_{a_{k}}^{\dagger} a_{i_{k}} \ldots a_{i_{1}} .
$$


Here and in the following, the indices $i, j, k, \ldots$ label occupied single-particle orbitals while $a, b, c, \ldots$ label unoccupied orbitals. The most commonly used approximation is coupled-cluster with singles-and-doubles excitations (CCSD) where $T \approx T_{1}+T_{2}$. The unknown amplitudes $t_{i}^{a}$ and $t_{i j}^{a b}$ in Eq. (2) are determined from the solution of the coupled-cluster equations

$$
\begin{aligned}
& 0=\left\langle\phi_{i}^{a}|\bar{H}| \phi_{0}\right\rangle, \\
& 0=\left\langle\phi_{i j}^{a b}|\bar{H}| \phi_{0}\right\rangle .
\end{aligned}
$$

Here $\left|\phi_{i}^{a}\right\rangle=a_{a}^{\dagger} a_{i}\left|\phi_{0}\right\rangle$ is a $1 \mathrm{p}$ - $1 \mathrm{~h}$ excitation of the reference state, and $\left|\phi_{i j}^{a b}\right\rangle$ is a similarly defined $2 \mathrm{p}-2 \mathrm{~h}$ excited state. The CCSD equations (4) thus demand that the reference state $\left|\phi_{0}\right\rangle$ has no $1 \mathrm{p}-1 \mathrm{~h}$ and no $2 \mathrm{p}-2 \mathrm{~h}$ excitations, i.e., it is an eigenstate of the similarity-transformed Hamiltonian (1) in the space of all $1 \mathrm{p}-1 \mathrm{~h}$ and $2 \mathrm{p}-2 \mathrm{~h}$ excited states. Once the CCSD equations are solved, the ground-state energy is computed as

$$
E=\left\langle\phi_{0}|\bar{H}| \phi_{0}\right\rangle
$$

Coupled-cluster theory has the following virtues. First, the similarity-transformed Hamiltonian can be evaluated exactly since the Hausdorff-Baker-Campbell expansion

$$
\begin{aligned}
\bar{H}= & H+[H, T]+\frac{1}{2 !}[[H, T], T] \\
& +\frac{1}{3 !}[[[H, T], T], T]+\ldots \\
= & \left(H e^{T}\right)_{c}
\end{aligned}
$$

terminates exactly at fourfold-nested commutators for two-body Hamiltonians. Here, the last term in parentheses, $(\ldots)_{c}$, indicates that only those terms enter where a cluster operator $T$ is connected to the Hamiltonian $H$ to the right. Since every cluster operator is linked to the Hamiltonian, coupled-cluster theory by construction fulfills Goldstone's linked cluster theorem and yields size-extensive results. This is particularly important in applications to medium-mass nuclei. Second, within the CCSD approximation, the computational effort scales as $n_{o}^{2} n_{u}^{4}$ where $n_{o}$ and $n_{u}$ denote the number of occupied and unoccupied orbitals in the reference state $\left|\phi_{0}\right\rangle$, respectively. Thus, the computational effort is much smaller than within the configuration interaction approach (or shell-model in nuclear physics) which exhibits a factorial scaling as function of the chosen single-particle space and number of nucleons.

Coupled-cluster theory with inclusion of full triples (CCSDT) [40] is usually considered to be too computationally expensive in most many-body systems of considerable size. Therefore triples corrections are usually taken into account perturbatively using the non-iterative CCSD(T) approach described in Ref. [41]. Recently, a more sophisticated way of including the full triples known as the $\Lambda$-CCSD(T) approach, has been developed by Taube et al. [42, 43]. In the $\Lambda$-CCSD $(\mathrm{T})$ approach the left-eigenvector solution of the CCSD similaritytransformed Hamiltonian is utilized in the calculation of a non-iterative triples correction to the coupled-cluster ground state energy. The left eigenvalue problem is given by

$$
\langle\psi| \Lambda \bar{H}=E\langle\psi| \Lambda
$$

were $\Lambda$ denotes the de-excitation cluster operator

$$
\Lambda=1+\Lambda_{1}+\Lambda_{2}
$$

with

$$
\begin{aligned}
& \Lambda_{1}=\sum_{i, a} \lambda_{a}^{i} a_{a} a_{i}^{\dagger}, \\
& \Lambda_{2}=\frac{1}{4} \sum_{i, j, a, b} \lambda_{a b}^{i j} a_{b} a_{a} a_{i}^{\dagger} a_{j}^{\dagger} .
\end{aligned}
$$

The unknowns, $\lambda_{a}^{i}$ and $\lambda_{a b}^{i j}$, result from the ground-state solution of the left eigenvalue problem (8). They are utilized together with the cluster amplitudes, $t_{i}^{a}$ and $t_{i j}^{a b}$, to compute the energy correction due to triples clusters as

$$
\begin{aligned}
\Delta E_{3} & =\frac{1}{(3 !)^{2}} \sum_{i j k a b c}\left\langle\psi\left|\Lambda\left(F_{h p}+V\right)_{N}\right| \psi_{i j k}^{a b c}\right\rangle \\
& \times \frac{1}{\varepsilon_{i j k}^{a b c}}\left\langle\psi_{i j k}^{a b c}\left|\left(V_{N} T_{2}\right)_{C}\right| \psi\right\rangle .
\end{aligned}
$$

Here, $F_{h p}$ denotes the part of the normal-ordered onebody Hamiltonian that annihilates particles and creates holes, while

$$
\varepsilon_{i j k}^{a b c} \equiv f_{i i}+f_{j j}+f_{k k}-f_{a a}-f_{b b}-f_{c c}
$$

is expressed in terms of the diagonal matrix elements of the normal-ordered one-body Hamiltonian $F$. In the case of Hartree-Fock orbitals, the one-body part of the Hamiltonian is diagonal and $F_{h p}$ vanishes. The subscript $C$ denotes the connected part of the operator, and $\left|\psi_{i j k}^{a b c}\right\rangle$ is a $3 \mathrm{p}-3 \mathrm{~h}$ excitation of the reference state.

\section{B. Equation-of-motion coupled-cluster theory for ground and excited states of closed- and open-shell nuclei}

The CCSD and the $\Lambda$-CCSD $(\mathrm{T})$ approaches are known as single-reference coupled-cluster methods (SR-CCM), and therefore they are particularly well suited for nuclei with an expected closed (sub-) shell structure. By adding or removing particles to a closed-shell nucleus, we move into regions of nuclei with open-shell structure, and clearly the SR-CCM methods are not well suited for the description of these nuclei. Most nuclei are of open-shell character and in order to study and predict properties like the evolution of shell-structure as we move towards the drip line in various isotopic chains, we clearly need to go beyond the SR-CCM class of approaches. There exists a variety of coupled-cluster 
methods that have been specifically designed to address the structure of open-shell systems, see Ref. [33] for an overview of some of these methods. Most methods are either based on a multi-reference formulation of the coupled-cluster method (MR-CCM) (see Ref. 44] for a discussion of various SR-CCM and MR-CCM), or an extension of equation-of-motion (EOM) theory [45] based on the coupled-cluster method (EOM-CCM) (see, e.g., Refs. [46, 47]). The beauty of the EOM-CCM is that it has the simplicity and transparency of SR-CCM such as the CCSD and the $\Lambda$-CCSD $(\mathrm{T})$ approaches discussed above, and that the method allows for systematic improvements and extensions.

In order to extend our ab initio coupled-cluster program beyond closed (sub-) shell nuclei, we use the EOMCCM approach. We give a brief outline of the method in the following. The basic idea behind EOM [45] is to calculate states $\left|\psi_{k}\right\rangle$ of the nucleus $B$ by acting with an excitation operator $\Omega_{k}$ on the ground state $\left|\psi_{0}\right\rangle$ of a closed-shell reference nucleus $A$. If $\Omega_{k}$ is a particle number-conserving operator $(B=A), \Omega_{k}$ will generate excited states of the nucleus $A$. In the case of $\Omega_{k}$ not conserving the number of particles $(B \neq A), \Omega_{k}$ can generate ground and excited states of the open-shell nucleus $B$ with respect to the reference nucleus $A$. In EOM-CC theory, the ground state of the closed-shell reference nucleus $A$ is given by the coupled-cluster wave function, $\left|\psi_{0}\right\rangle=\exp (T)\left|\phi_{0}\right\rangle$. In case we wish to calculate excited states of the closed-shell nucleus $A(B=A)$, we define the excitation operator $\Omega_{k}=R_{k}^{A}$,

$$
R_{k}^{A}=r_{0}+\sum_{i, a} r_{i}^{a} a_{a}^{\dagger} a_{i}+\frac{1}{4} \sum_{i, j, a, b} r_{i j}^{a b} a_{a}^{\dagger} a_{b}^{\dagger} a_{j} a_{i}+\ldots
$$

and by truncating at the two-particle-two-hole excitation level, we get the standard EOM-CCSD approach. In case we wish to calculate ground and excited states of a nucleus with a nucleon added or removed from a closed-shell nucleus ( $B=A \pm 1$ ), we define the excitation operators $\Omega_{k}=R_{k}^{A \pm 1}$ with,

$$
\begin{array}{r}
R_{k}^{(A+1)}=\sum_{a} r^{a} a_{a}^{\dagger}+\frac{1}{2} \sum_{j, a, b} r_{j}^{a b} a_{a}^{\dagger} a_{b}^{\dagger} a_{j}+\ldots \\
R_{k}^{(A-1)}=\sum_{i} r_{i} a_{i}+\frac{1}{2} \sum_{i, j, a} r_{i j}^{a} a_{a}^{\dagger} a_{i} a_{j}+\ldots
\end{array}
$$

and truncating at the two-particle-one-hole and twohole-one-particle levels, we get the the particle-attached and particle-removed equation-of-motion coupled-cluster methods (PA/PR-EOM-CCM), see, for example, Refs. [33, 47]. The PA-EOM-CCM adds a particle to a nucleus with $A$ nucleons by creating $1 p, 2 p-1 h, 3 p-2 h, \ldots$, excitations on the ground state of the nucleus with $A$ nucleons. Similarly, PR-EOM-CCM removes a particle from $A$ by creating $1 h, 1 p-2 h, 2 p-3 h, \ldots$, excitations on the ground state of $A$. By multiplying the equation for the nucleus $A, \bar{H}\left|\phi_{0}\right\rangle=E_{0}\left|\phi_{0}\right\rangle$, from the left with $\Omega_{k}$, and subtracting it from the equation for the nucleus $B$, $\bar{H} \Omega_{k}\left|\phi_{0}\right\rangle=E_{k} \Omega_{k}\left|\phi_{0}\right\rangle$, we obtain the following equation,

$$
\left[\bar{H}, \Omega_{k}\right]\left|\phi_{0}\right\rangle=\left(\bar{H} \Omega_{k}\right)_{C}\left|\phi_{0}\right\rangle=\omega_{k} \Omega_{k}\left|\phi_{0}\right\rangle,
$$

with $\bar{H}=e^{-T} H e^{T}$ being the similarity-transformed Hamiltonian and $\omega_{k}=E_{k}-E_{0}$ the energy of the nucleus $B$ relative to the ground state of the nucleus $A$. Eq. (16) defines a right eigenvalue problem for the excitation amplitudes in Eqs. (14) and (15), and is usually solved using iterative eigenvalue algorithms.

\section{Spherical coupled-cluster theory}

For "hard" interactions such as the "bare" $\mathrm{N}^{3} \mathrm{LO}$ interaction, wave function methods need to employ very large model spaces in order to yield converged results. For spherical reference states (nuclei with closed major shells or closed subshells), it is therefore better to employ the spherical symmetry to further reduce the number of unknowns, that is, the number of cluster amplitudes. For such nuclei, the cluster operator of Eq. (2) is a scalar under rotation, and depends only on reduced amplitudes. Thus,

$$
T_{1}=\sum_{j_{i} j_{a}} t_{j_{i}}^{j^{a}}\left(a_{j_{a}}^{\dagger} \times \tilde{a}_{j_{i}}\right)^{(0)}
$$

and

$$
T_{2}=\sum_{j_{i} j_{j} j_{a} j_{b} J} t_{j_{i} j_{j}}^{j_{a} j_{b}}(J)\left(a_{j_{a}}^{\dagger} \times a_{j_{b}}^{\dagger}\right)^{(J)} \cdot\left(\tilde{a}_{j_{j}} \times \tilde{a}_{j_{i}}\right)^{(J)} .
$$

Here, we employed the usual notation for spherical tensors, and $j_{i}$ and $j_{a}$ denote the spin of the occupied and unoccupied subshells, respectively. It is clear that the similarity-transformed Hamiltonian is also a scalar under rotation, and it is straightforward to work out the CCSD equations within this formulation. Details are given in the Appendix. The computational cost of PAEOM-CCSD is like that of CCSD, i.e., $n_{u}^{4} n_{o}^{2}$, where $n_{u}$ is the number of unoccupied orbitals and $n_{o}$ is the number of occupied orbitals. In order to reduce the memory and computational cost related to the basis size, we write the excitation amplitudes $R_{k}^{(A \pm 1)}$ as spherical tensors of rank $J$ and $\operatorname{projection} M$,

$$
\begin{aligned}
& R_{k}^{(A+1)}(J, M)=\sum_{j_{a}} r^{j_{a}}(J) a_{j_{a} m_{a}}^{\dagger} \delta_{J}^{j_{a}} \delta_{M}^{m_{a}} \\
& +\frac{1}{2} \sum_{j_{j} j_{a} j_{b} J_{a b}} r_{j_{j}}^{j_{a} j_{b}}\left(J_{a b}, J\right)\left[\left(a_{j_{a}}^{\dagger} \otimes a_{j_{b}}^{\dagger}\right)^{J_{a b}} \otimes \tilde{a}_{j_{j}}\right]_{M}^{(J)}, \\
& R_{k}^{(A-1)}(J, M)=\sum_{j_{i}} r^{j_{i}}(J) a_{j_{i} m_{i}} \delta_{J}^{j_{i}} \delta_{M}^{m_{i}} \\
& \quad+\frac{1}{2} \sum_{j_{i} j_{j} j_{a} J_{i j}} r_{j_{i} j_{j}}^{j_{a}}\left(J_{i j}, J\right)\left[a_{j_{a}}^{\dagger} \otimes\left(\tilde{a}_{j_{i}} \otimes \tilde{a}_{j_{j}}\right)^{J_{i j}}\right]_{M}^{(J)} .
\end{aligned}
$$


Here, we wish to solve for the reduced excitation amplitudes $r^{j_{a}}(J)$ and $r_{j_{j}}^{j_{a}, j_{b}}\left(J_{a b}, J\right)$. In this coupled scheme, the eigenvalue equation in Eq. (16) is solved separately for each set of quantum numbers $\left\{J^{\pi}, T_{z}\right\}$.

A simple estimate shows that a model space of $n_{o}+n_{u}$ single-particle states consists of only $\left(n_{o}+n_{u}\right)^{2 / 3} j$-shells. Thus, the entire computational effort is approximately reduced by a power $2 / 3$ within the spherical scheme compared to the $m$-scheme. We have derived and numerically implemented the spherical scheme within the CCSD approximation. We tested that our $m$-scheme code and the spherical code give identical results for several test cases. The spherical code permits us to reach much larger model spaces, and we are able to achieve satisfactory convergence even for "hard" interactions such as the "bare" $\mathrm{N}^{3} \mathrm{LO}$ interaction. In Ref. [8] we used the spherical coupled-cluster code to calculate the ground states of medium-mass nuclei like ${ }^{40,48} \mathrm{Ca}$ and ${ }^{48} \mathrm{Ni}$ within the CCSD approximation and starting from bare chiral interactions. In a model space of 15 major oscillator shells, the results were reasonably well converged. Recently, we have also implemented the $\Lambda$ - $\operatorname{CCSD}(\mathrm{T})$ approach in an angular-momentum-coupled scheme. In Ref. [27], we calculated the ground states of the oxygen isotopes using chiral interactions in model spaces comprising up to 20 major oscillator shells within the $\Lambda$-CCSD(T) approach. In such large spaces we were even able to converge the ground state energies for a chiral interaction with a $600 \mathrm{MeV} c^{-1}$ momentum cutoff. Likewise, the inclusion of continuum scattering states - necessary for a description of halo states in weakly bound nuclei - yields large model spaces that can be treated within he spherical scheme [18]. Details of the angular-momentum-coupled $\Lambda$-CCSD $(\mathrm{T})$ approach are given in the Appendix.

\section{Expectation values in coupled-cluster theory}

For expectation values and the computation of ground state properties other than the energy, we utilize the right and left eigenvectors of the similarity-transformed Hamiltonian $\bar{H}$ and compute the one- and two-body reduced density matrices,

$$
\begin{array}{r}
\rho_{p q}=\left\langle\phi_{0}\left|\Lambda e^{-T} a_{p}^{\dagger} a_{q} e^{T}\right| \phi_{0}\right\rangle, \\
\rho_{p q r s}=\left\langle\phi_{0}\left|\Lambda e^{-T} a_{p}^{\dagger} a_{q}^{\dagger} a_{r} a_{s} e^{T}\right| \phi_{0}\right\rangle .
\end{array}
$$

Expectation values can then be computed by expressing the operator of interest in terms of the density matrices. The Hellmann-Feynman theorem is another route to the computation of expectation values. In this case, we consider a response of the ground-state energy to a perturbation caused by a given operator of interest. The Hellmann-Feynman theorem expresses the expectation values of an observable $B$ as

$$
\langle B\rangle=\left.\frac{\partial E(\beta)}{\partial \beta}\right|_{\beta}=0 .
$$

Here $E(\beta)$ is the ground-state energy of the Hamiltonian $H+\beta B$. Unfortunately, the relation (23) does not hold exactly within the coupled-cluster approach since this method is not variational when the cluster operator is truncated. We will nevertheless base the computation of expectation values on Eq. (23). Experience shows that this approach is approximately valid if the relevant particle-hole excitations are incorporated. In practice, the differential quotient (23) is numerically implemented as a difference quotient with $\beta \approx 0.01$. This requires us to perform two calculations (one for zero and one for small nonzero $\beta$ ). For relatively "hard" interactions such as the "bare" $\mathrm{N}^{3} \mathrm{LO}$, we also perform two Hartree-Fock calculations and thus employ slightly different single-particle bases in these two cases. While such a procedure is unnecessary for methods that fulfill the Hellmann-Feynman theorem, it is important within our approach. We note that this approach most closely reflects the physical situation of an expectation value measuring the system's response to a perturbation

\section{TREATMENT OF THE CENTER-OF-MASS PROBLEM}

In this section, we demonstrate that the coupledcluster wave function factorizes to a very good approximation into a product of a center-of-mass wave function and an intrinsic wave function. We will present a simple procedure that checks this factorization and give an estimate for the degree of the achieved factorization. This section significantly expands on the short demonstration of the factorization in Ref. [26].

\section{A. Statement of the center-of-mass problem}

Let us consider the nuclear $A$-body Hamiltonian

$$
H_{A}=\sum_{j=1}^{A} \frac{\vec{p}^{2}}{2 m}+\sum_{j<k}^{A} V(j, k) .
$$

Here, $V$ is a two-body operator that is invariant under rotations and translations. Thus, the total momentum and angular momentum are conserved quantities. It is advantageous to separate the Hamiltonian into an intrinsic Hamiltonian $H_{\text {in }}$ and the center-of-mass Hamiltonian (i.e., the kinetic energy $T_{\mathrm{cm}}$ of the center of mass) as

$$
\begin{aligned}
H & =T_{\mathrm{cm}}+H_{\mathrm{in}} \\
& =T_{\mathrm{cm}}+\sum_{j<k}^{A}\left(\frac{\left(\vec{p}_{j}-\vec{p}_{k}\right)^{2}}{2 m A}+V(j, k)\right) .
\end{aligned}
$$

Note that the intrinsic Hamiltonian $H_{\text {in }}$ does not depend on the center-of-mass coordinate. Thus, we could add an arbitrary operator $H_{\mathrm{cm}}$ of the center-of-mass coordinate to the intrinsic Hamiltonian $H_{\text {in }}$ without changing the 
intrinsic properties of the resulting Hamiltonian. In other words, the eigenfunctions $\psi_{A}$ of the $A$-body Hamiltonian $H_{\text {in }}+H_{\text {cm }}$ are products of an intrinsic eigenfunction $\psi_{\text {in }}$ of the intrinsic Hamiltonian $H_{\text {in }}$, and a center-of-mass wave function $\psi_{\mathrm{cm}}$ that is the eigenfunction of the center-ofmass Hamiltonian $H_{\mathrm{cm}}$, that is,

$$
\psi_{A}=\psi_{\mathrm{cm}} \psi_{\mathrm{in}}
$$

The corresponding energy is the sum $E_{A}=E_{\mathrm{cm}}+E_{\text {in }}$ of the center-of-mass energy and the intrinsic energy. The question thus arises which operator $H_{\mathrm{cm}}$ to choose. Let us consider the NCSM [7] as an example. Here, one works in a complete $N \hbar \omega$ space consisting of a basis of all $A$ particle Slater determinants of oscillator states with frequency $\omega$ and total excitation energy not exceeding $N \hbar \omega$. One can add the center-of-mass Hamiltonian

$$
H_{\mathrm{cm}}(\tilde{\omega})=T_{\mathrm{cm}}+\frac{1}{2} m A \tilde{\omega}^{2} R_{\mathrm{cm}}^{2}-\frac{3}{2} \hbar \tilde{\omega}
$$

with $\tilde{\omega}=\omega$ to the intrinsic Hamiltonian $H_{\mathrm{in}}$, and obtains a factorized ground-state wave function where the center-of-mass wave function $\psi_{\mathrm{cm}}$ is a Gaussian with frequency $\omega$. The (truncated) coupled-cluster method is unable to employ an $N \hbar \omega$ space, and it is arguably the best idea to completely remove any reference to the centerof-mass coordinate. Thus, we solve an intrinsic Hamiltonian $H_{\text {in }}$ that depends on $A-1$ independent coordinates in a Hilbert space spanned by wave functions of $A$ coordinates. Two comments are in order. First, the truncated coupled-cluster method is not an exact solution of the $A$-body problem, but rather a very efficient approximation. Thus, it is not guaranteed a priori that the coupled-cluster wave function exhibits the factorization (26). Recall that any $A$-body wave function can be expanded as

$$
\psi_{A}=\sum_{j \geq 1} s_{j} \psi_{\mathrm{cm}}^{(j)} \psi_{\mathrm{in}}^{(j)}
$$

where $\sum_{j} s_{j}^{2}=1$ from normalization, and we assume that the non-negative weights $s_{j}$ are ordered in decreasing order. Only if all but one of the weights $s_{j}$ vanishes, does the factorization (26) take place; otherwise, the factorization might be mildly or strongly violated, depending on the size of the weights $s_{j}$. Second, if a factorization of the coupled-cluster wave function takes (approximately) place, the form of the center-of-mass wave function $\psi_{\mathrm{cm}}^{(1)}$ corresponding to the largest weight $s_{1}$ has to be determined. In what follows we will see that the $A$ body coupled-cluster wave function factorizes to a good approximation (i.e., $s_{1} \approx 1$ ), and that the center-of-mass wave function is the Gaussian ground state of the centerof-mass Hamiltonian (27) for a yet-to-be determined frequency $\tilde{\omega}$.

\section{B. Approximate separation of the center-of-mass wave function in coupled-cluster calculations}

We consider the nucleus ${ }^{16} \mathrm{O}$ and employ the lowmomentum interaction $V_{\text {low } k}$ with a smooth momentum cutoff $\lambda=1.8 \mathrm{fm}^{-1}$ derived from Machleidt's and Entem's chiral $\mathrm{N}^{3} \mathrm{LO}$ interaction. Figure 1 shows the ground-state energy as a function of the oscillator spacing $\hbar \omega$ of the underlying oscillator basis in a model space of nine oscillator shells. These results are obtained from a CCSD calculation within a spherical Hartree-Fock basis. The energy is well converged as it depends very weakly on the model-space parameters. We also computed the ground-state expectation value

$$
E_{\mathrm{cm}}(\tilde{\omega})=\langle H(\tilde{\omega})\rangle
$$

of the center-of-mass Hamiltonian (27) for a frequency $\tilde{\omega}=\omega$ and show the result in the inset of Fig. 11 This expectation value is generally not zero. This indicates that the coupled-cluster wave function is in general not an eigenstate of the center-of-mass Hamiltonian $H(\omega)$. However, there seems to be little correlation between the ground-state energy and the expectation value $E(\omega)$, and the latter vanishes approximately in a model space with $\hbar \omega \approx 20 \mathrm{MeV}$. Thus, at this frequency, the coupledcluster wave function is approximately the ground state of the center-of-mass Hamiltonian $H(\omega)$. As a check, we fix $\hbar \omega=20 \mathrm{MeV}$, consider the Hamiltonian $H=$ $H_{\text {in }}+\beta H(\omega)$, and compute its ground-state energy as a function of the parameter $\beta$. The result is shown in Fig. 2. Clearly, the ground-state energy is rather insensitive to $\beta$ and varies by only $15 \mathrm{keV}$ as $\beta$ is increased from zero to one.

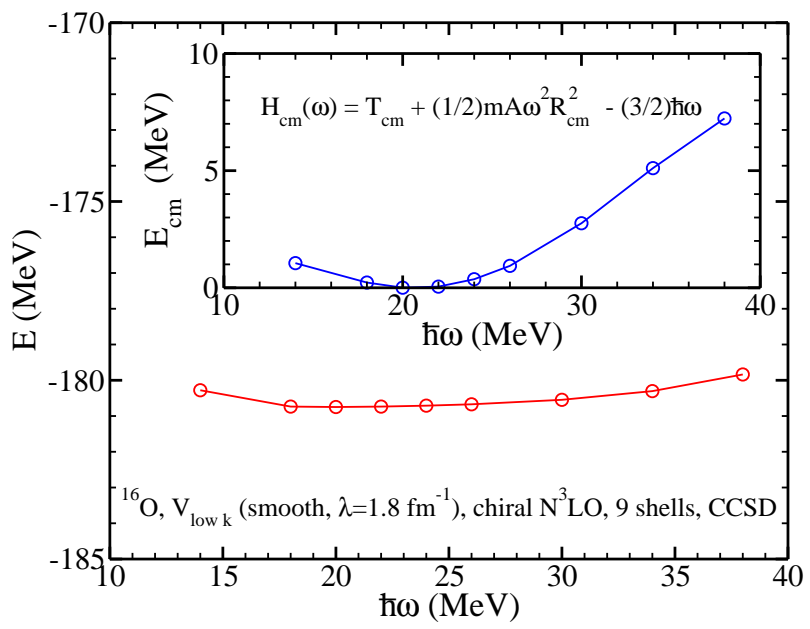

FIG. 1: (Color online) Ground-state energy (within CCSD) of ${ }^{16} \mathrm{O}$ with a low-momentum interaction as a function of the oscillator spacing $\hbar \omega$. The model space consists of nine major oscillator shells. Inset: Expectation value $E_{\mathrm{cm}}(\omega)$ of the center-of mass Hamiltonian with the standard frequency dependence. 


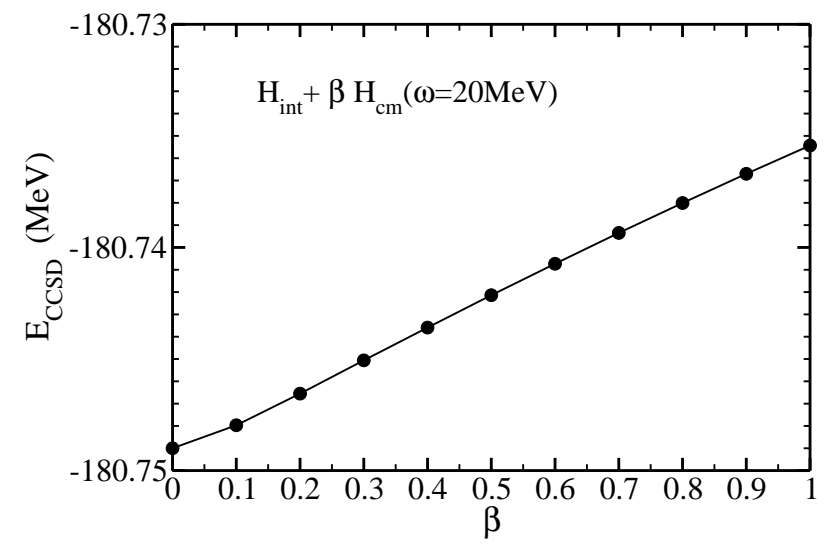

FIG. 2: (Color online) Ground-state energy (within CCSD) of ${ }^{16} \mathrm{O}$ using a low-momentum interaction with the centerof-mass Hamiltonian $\beta H_{\mathrm{cm}}$ added. Calculations employ nine major oscillator shells and $\hbar \omega=20 \mathrm{MeV}$. As $\beta$ is varied between 0 and 1 , the ground state energy changes by about $15 \mathrm{keV}$.

The factorization of Eq. (26) is thus achieved in a model space with $\hbar \omega \approx 20 \mathrm{MeV}$. Let us assume that such a factorization takes place generally, and that the corresponding center-of-mass wave function is a Gaussian with unknown frequency $\tilde{\omega}$ that might differ from the frequency of the underlying oscillator basis. Thus, we assume that the coupled-cluster wave function is the ground state of the center-of-mass Hamiltonian (27) for a suitable frequency $\tilde{\omega}$. To determine this frequency we employ the identity

$H_{\mathrm{cm}}(\omega)+\frac{3}{2} \hbar \omega-T_{\mathrm{cm}}=\frac{\omega^{2}}{\tilde{\omega}^{2}}\left(H_{\mathrm{cm}}(\tilde{\omega})+\frac{3}{2} \hbar \tilde{\omega}-T_{\mathrm{cm}}\right)$,

and take its expectation value. We seek (and insert) $E_{\mathrm{cm}}(\tilde{\omega})=0$, employ the relation $\left\langle T_{\mathrm{cm}}\right\rangle=\frac{3}{4} \hbar \tilde{\omega}$ valid for Gaussians, insert the already computed expectation values $E_{\mathrm{cm}}(\omega)$, and solve for the unknown frequency $\tilde{\omega}$. This yields the two possible solutions.

$$
\begin{aligned}
\hbar \tilde{\omega}= & \hbar \omega+\frac{2}{3} E_{\mathrm{cm}}(\omega) \\
& \pm \sqrt{\frac{4}{9}\left(E_{\mathrm{cm}}(\omega)\right)^{2}+\frac{4}{3} \hbar \omega E_{\mathrm{cm}}(\omega)} .
\end{aligned}
$$

We compute the ground-state expectation values $E_{\mathrm{cm}}(\tilde{\omega})$ for these two frequencies and find that one expectation value is typically very close to zero. Figure 3 shows that the small expectation value essentially vanishes for a large range of frequencies of the underlying oscillator basis in a model space of 13 major oscillator shells. Closer inspection shows that the expectation value is about $E_{\mathrm{cm}}(\tilde{\omega}) \approx-10 \mathrm{keV}$. Recall that coupled-cluster theory is non-variational (as the similarity-transformed Hamiltonian is non-Hermitian), and such a small negative expectation value is certainly tolerable for the non-negative operator (27). Figure 3 also shows that the frequency of

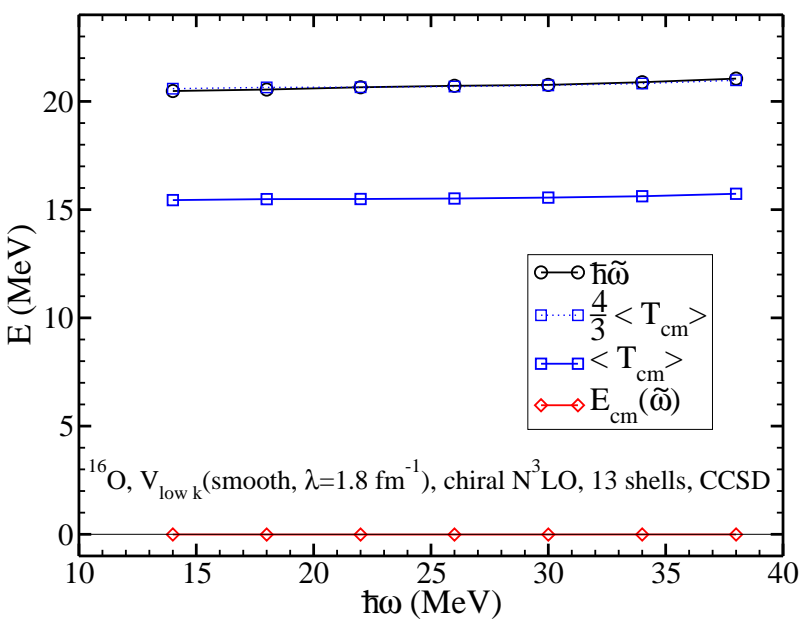

FIG. 3: (Color online) ${ }^{16} \mathrm{O}$ Ground-state expectation value (within CCSD) of the generalized center-of-mass Hamiltonian $H_{\mathrm{cm}}(\tilde{\omega})$ and of the kinetic energy $T_{\mathrm{cm}}$ as a function of the oscillator spacing $\hbar \omega$. The model space consists of thirteen major oscillator shells.

the Gaussian center-of-mass wave function stays approximately constant $\hbar \tilde{\omega} \approx 20 \mathrm{MeV}$ for a large range of frequencies $\omega$ of the underlying oscillator basis. As a final check, we also computed the ground-state expectation value of the center-of-mass kinetic energy. For a Gaussian, this expectation value fulfills $\left\langle T_{\mathrm{cm}}\right\rangle=\frac{3}{4} \hbar \tilde{\omega}$. Figure 3 shows that this relation is indeed obeyed.

Let us turn to the "bare" $\mathrm{N}^{3} \mathrm{LO}$ interaction [13, 15]. This interaction has a substantially higher momentum cutoff than the previously employed low-momentum interaction. For converged results, we need to employ larger model spaces and also triples clusters. We performed coupled-cluster calculations of the ground states of ${ }^{16} \mathrm{O}$ and ${ }^{4} \mathrm{He}$. Figure 4 shows the ground-state energy of ${ }^{16} \mathrm{O}$ (lower panel), the ground-state expectation value $E_{\mathrm{cm}}(\tilde{\omega})$ (upper panel) of the center-of-mass Hamiltonian (27), and the corresponding frequency $\tilde{\omega}$ (middle panel) as a function of the frequency $\omega$ of the underlying oscillator basis. One sees that the ground-state energy is well converged and displays only a weak dependence on $\omega$. Similar comments apply to the frequency $\tilde{\omega}$. The expectation value $E_{\mathrm{cm}}(\tilde{\omega})$ is small, i.e., $\left|E_{\mathrm{cm}}(\tilde{\omega})\right| \ll \hbar \tilde{\omega}$ but nonzero. For smaller values of the frequency $\omega$ of the underlying oscillator basis, we are at the limit of wellconverged results, and $E_{\mathrm{cm}}$ even becomes negative. This shows that the coupled-cluster ground state is not an exact eigenstate of the center-of-mass Hamiltonian (27). Let us also assume that the factorization is not perfect (though this is not implied by a nonzero value of $E_{\mathrm{cm}}$ ). We want to estimate the level of admixture of centerof-mass excitations. A simple and conservative estimate based on perturbation theory shows that the admixture is essentially $E_{\mathrm{cm}} / \hbar \tilde{\omega} \approx 5 \%$. We also computed the in- 


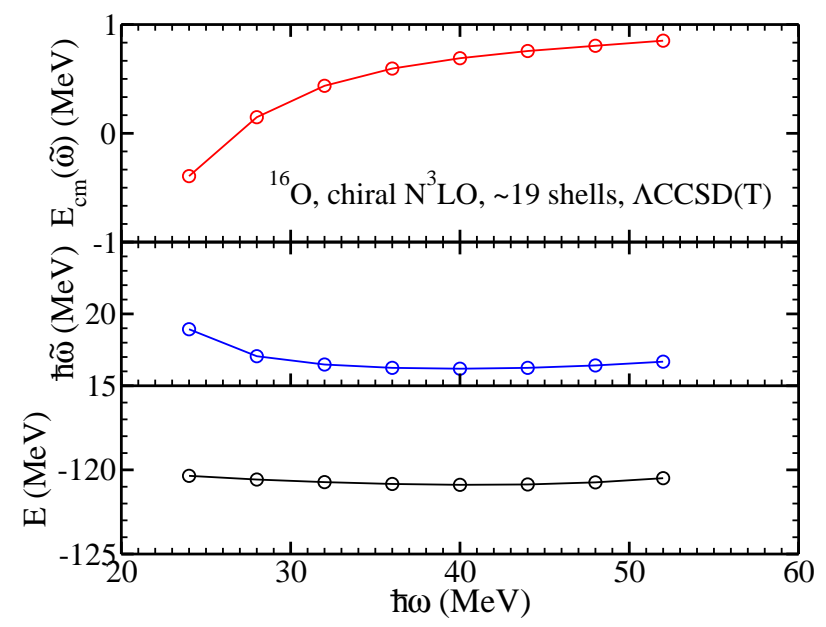

FIG. 4: (Color online) Bottom: Ground-state energy of ${ }^{16} \mathrm{O}$ within the $\Lambda$-CCSD $(\mathrm{T})$ approximation as a function of the frequency $\hbar \omega$ of the underlying oscillator basis. Middle: Relation between the frequency $\tilde{\omega}$ and the frequency $\omega$ of the underlying oscillator basis. Top: Expectation value $E_{\mathrm{cm}}(\tilde{\omega})$ of the center-of-mass vs. $\hbar \omega$.

trinsic point radius $r$ from

$$
r^{2} \equiv \frac{1}{A^{2}} \sum_{1 \leq j<k \leq A}\left\langle\left(\vec{r}_{j}-\vec{r}_{k}\right)^{2}\right\rangle
$$

and show the result in Fig. 5 . This calculation is again based on the triples-corrected $\Lambda$-CCSD $(\mathrm{T})$ approximation, and it still exhibits a weak model-space dependence. It provides us with a further test of the wave-function factorization, since we can employ the center-of-mass coordinate $\vec{R}_{\mathrm{cm}}$ and rewrite Eq. (31) as

$$
r^{2}=\frac{1}{A} \sum_{j=1}^{A}\left\langle\left(\vec{r}_{j}-\vec{R}_{\mathrm{cm}}\right)^{2}\right\rangle=\frac{1}{A} \sum_{j=1}^{A}\left\langle\vec{r}_{j}^{2}\right\rangle-\left\langle\vec{R}_{\mathrm{cm}}^{2}\right\rangle .
$$

For a Gaussian corresponding to the frequency $\tilde{\omega}$, we have $\frac{1}{2} m A \tilde{\omega}^{2}\left\langle\vec{R}_{\mathrm{cm}}^{2}\right\rangle=\frac{3}{4} \hbar \tilde{\omega}$, and the intrinsic point radius can thus be computed from the expectation value of a onebody operator. The result from this calculation is in very good agreement with the result obtained from Eq. (31), as shown in Fig. [5 This suggests that the factorization of the wave function might be better than expected from the calculation of the ground-state expectation value (29) depicted in Fig. 4.

At the moment, we have no analytical insights into the observed factorization and the Gaussian shape of the center-of-mass wave function. We believe that the factorization itself is not entirely surprising. While our finite basis is not complete in a mathematical sense, it is sufficiently complete to describe low-energy nuclear structure, i.e., it contains momentum modes that exceed the cutoff of the interaction, and it is sufficiently extended in position space to accommodate a quantum object of the size of the nucleus. Thus, one expects the basis to capture

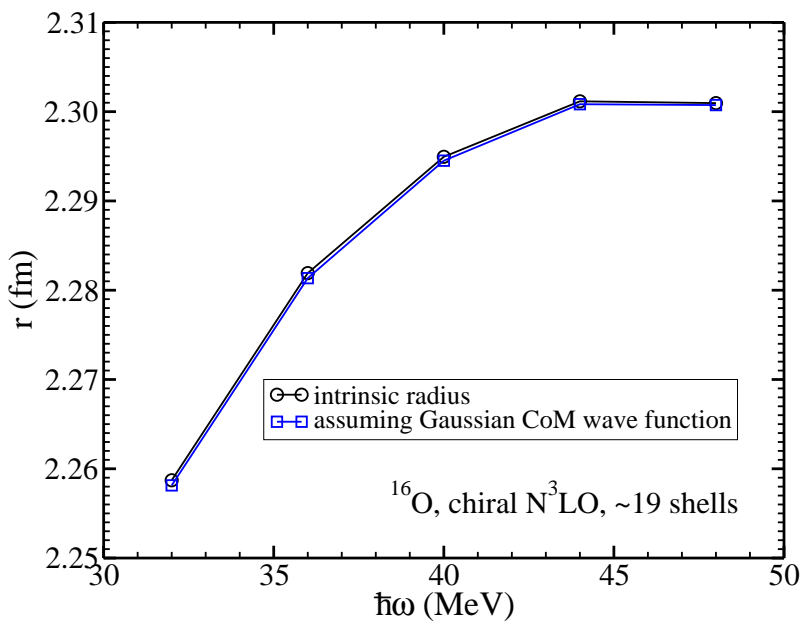

FIG. 5: (Color online) RMS radii of ${ }^{16} \mathrm{O}$ within the $\Lambda$ $\operatorname{CCSD}(\mathrm{T})$ approximation using the intrinsic point radius and subtracting $\left\langle R_{\mathrm{cm}}^{2}\right\rangle$ assuming a Gaussian for the center of mass.

the relevant physics, and a well-factorized ground state is not too surprising, even if the employed many-body basis functions do not individually reflect this factorization.

\section{Center-of-mass problem for open-shell nuclei}

Odd-mass nuclei that differ from a nucleus with closed subshells by the addition or removal of one nucleon can also be computed efficiently within the coupled-cluster method. For the calculation of ${ }^{17} \mathrm{O}$, we employ the particle-attached equation-of-motion method (16), which is based on describing ${ }^{17} \mathrm{O}$ as a nucleon (i.e a superposition of $1 \mathrm{p}$ and $2 \mathrm{p}-1 \mathrm{~h}$ excitations) upon the ground state of ${ }^{16} \mathrm{O}$. We first solve the coupled-cluster equations (4) for the "mass-shifted" nucleus ${ }^{16} \mathrm{O}$, i.e., for the computation of the ${ }^{16} \mathrm{O}$ ground state, we employ the Hamiltonian (24) with mass number $A=17$. In a second step, we describe the ground and excited states in ${ }^{17} \mathrm{O}$ in terms of the excitations (15) upon this ${ }^{16} \mathrm{O}$ ground state. For an SRG interaction with cutoff $\lambda=2.8 \mathrm{fm}^{-1}$, the energies of a few low-lying states are shown in Fig. [ as a function of the oscillator frequency in a model space with $N=12$. These states are single-particle states and can be well computed within our approach. Figure 7 shows the energy expectation value $E_{\mathrm{cm}}(\omega)$ (defined in Eq. (29)) for the three low-lying single-particle states as a function of the oscillator frequency $\omega$ of the underlying model space. These expectation values are small for $\omega \approx 16 \mathrm{MeV}$, but large for other parameters. This shows that the centerof-mass wave function is generally not a Gaussian with frequency $\omega$. No further conclusion can be reached from this expectation value. In particular, this is no evidence that the center-of-mass wave function does not separate.

Let us again assume that the coupled-cluster wave function factorizes into a Gaussian (with frequency $\tilde{\omega}$ ) 


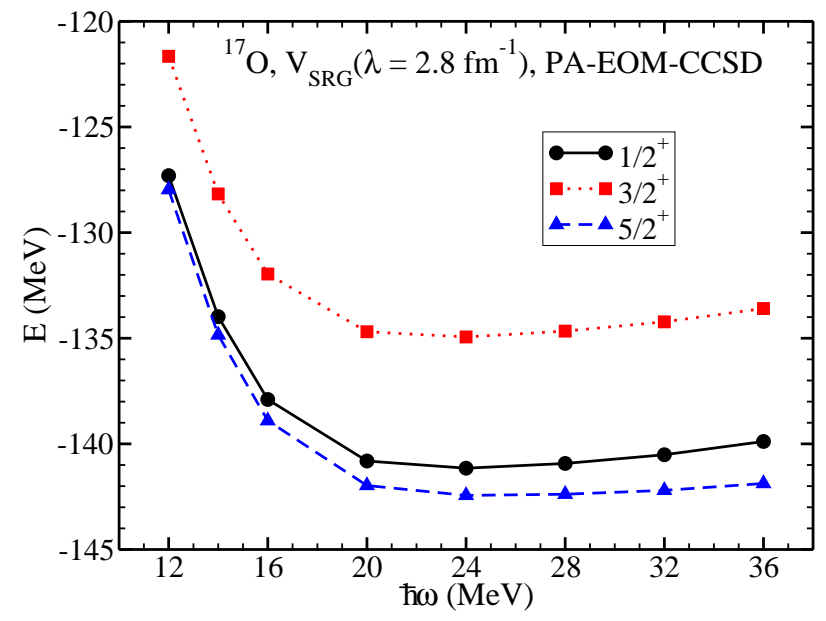

FIG. 6: (Color online) Energies of low-lying $J^{\pi}=1 / 2^{+}, J^{\pi}=$ $3 / 2^{+}, J^{\pi}=5 / 2^{+}$states in ${ }^{17} \mathrm{O}$ based on an SRG interaction with cutoff $\lambda=2.8 \mathrm{fm}^{-1}$ in a model space consisting of $N+$ $1=13$ oscillator shells versus the oscillator frequency.

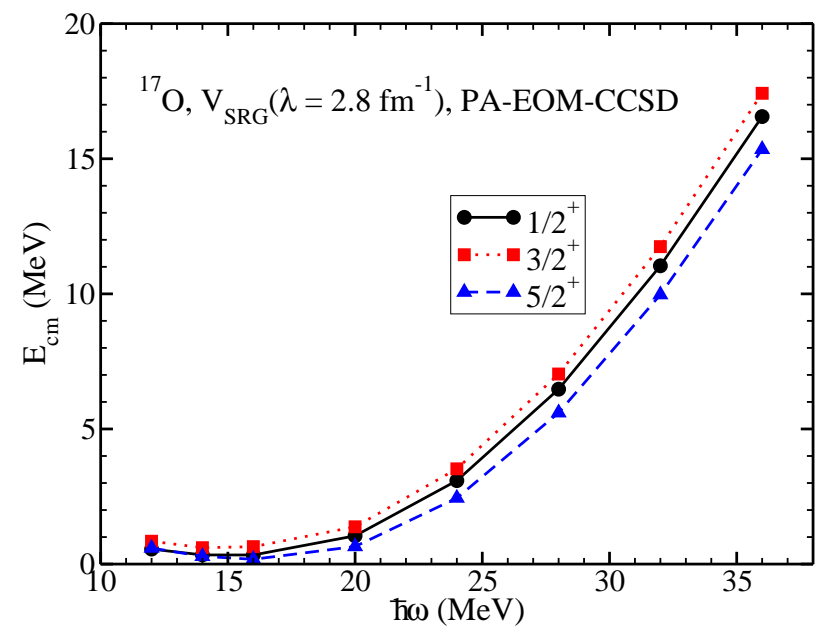

FIG. 7: (Color online) Expectation values $E_{\mathrm{cm}}(\omega)$ of the center-of-mass Hamiltonian $H_{\mathrm{cm}}(\omega)$ (see Eq. (27) ) for the lowlying $J^{\pi}=1 / 2^{+}, J^{\pi}=3 / 2^{+}, J^{\pi}=5 / 2^{+}$states in ${ }^{17} \mathrm{O}$ versus the oscillator frequency.

for the center of mass and an intrinsic wave function, and let us determine $\tilde{\omega}$ from Eq. (30). Figure 8 shows the resulting frequency for the three states we computed in ${ }^{17} \mathrm{O}$. The corresponding expectation values $E_{\mathrm{cm}}(\tilde{\omega})$ defined in Eq. (29) are shown in Fig. 9 as a function of the oscillator frequency $\omega$ of the underlying model space. We employ $\tilde{\omega}=\tilde{\omega}(\omega)$ as depicted in Fig. 8. As evident from Figs. 8 and 9 we have $E_{\mathrm{cm}}(\tilde{\omega}) \ll \hbar \tilde{\omega}$. Thus, the computed states in ${ }^{17} \mathrm{O}$ also exhibit a Gaussian center-ofmass wave function to a very good approximation (admixtures of higher $\hbar \tilde{\omega}$ oscillator excitations are of the order of $\left.E_{\mathrm{cm}}(\tilde{\omega}) / \hbar \tilde{\omega} \approx 1-2 \%\right)$. This implies that the factorization is at least of the same degree of quality.

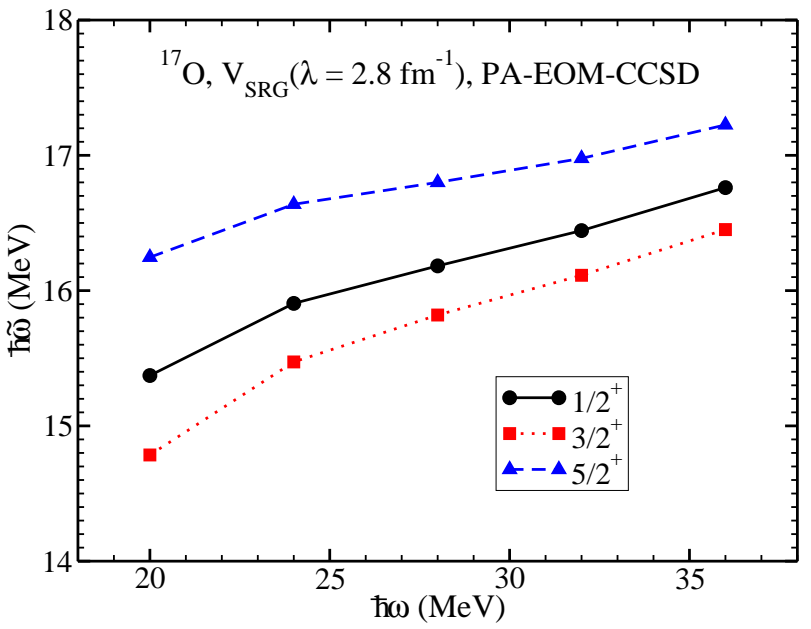

FIG. 8: (Color online) Frequency $\tilde{\omega}$ of the (approximately) Gaussian center-of-mass wave function for the low-lying $J^{\pi}=$ $1 / 2^{+}, J^{\pi}=3 / 2^{+}, J^{\pi}=5 / 2^{+}$states in ${ }^{17} \mathrm{O}$ versus the oscillator frequency.

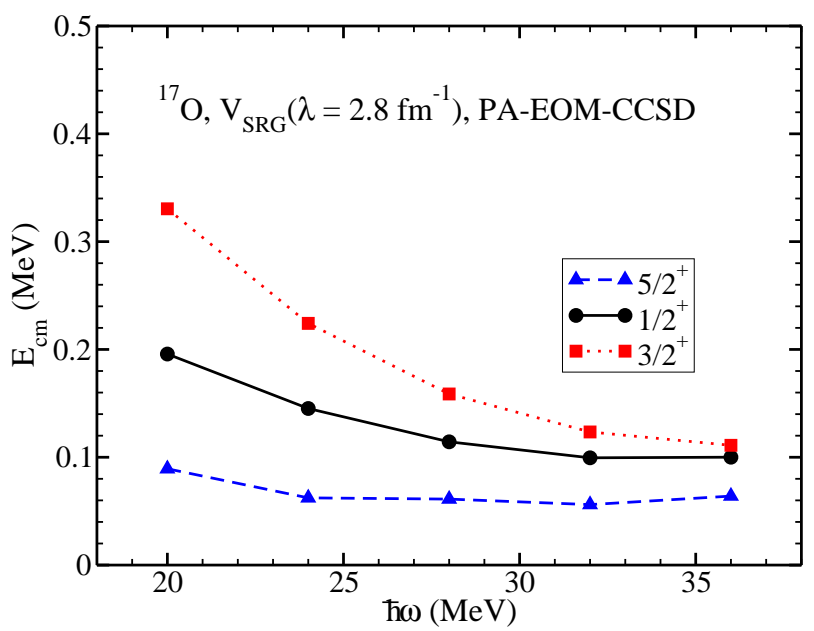

FIG. 9: (Color online) Expectation values $E_{\mathrm{cm}}(\tilde{\omega})$ of the center-of-mass Hamiltonian $H_{\mathrm{cm}}(\tilde{\omega})$ (see Eq. (27)) for the lowlying $J^{\pi}=1 / 2^{+}, J^{\pi}=3 / 2^{+}, J^{\pi}=5 / 2^{+}$states in ${ }^{17} \mathrm{O}$ versus the oscillator frequency. Here, the frequency $\tilde{\omega}=\tilde{\omega}(\omega)$ employed in the center-of-mass Hamiltonian is as depicted in Fig. 8 We have $E_{\mathrm{cm}}(\tilde{\omega}) \ll \hbar \tilde{\omega}$ and thus a very good separation of center-of-mass excitations.

\section{Center-of-mass problem in a toy model}

To shed some light onto the observed factorization, we consider a simple problem of two interacting particles in one spatial dimension. We choose a two-body interaction of the form $V(x)=-V_{0} \exp \left(-(x / l)^{2}\right)$, where $x=\left(x_{1}-\right.$ $\left.x_{2}\right) / \sqrt{2}$ is (up to a factor $\sqrt{2}$ ) the relative coordinate of the two-particle system, and $l$ is a length scale. We thus consider the intrinsic Hamiltonian

$$
H=\frac{p^{2}}{2 m}-V_{0} \exp \left(-(x / l)^{2}\right)
$$


with $m$ being the mass and $p$ the relative momentum $p=\left(p_{1}-p_{2}\right) / \sqrt{2}$. We choose a basis consisting of products $\Phi_{m}\left(x_{1} / l\right) \Phi_{n}\left(x_{2} / l\right)$ of oscillator wave functions $\Phi_{k}$, and choose $0 \leq m, n \leq N$. Thus, the basis is not a complete $N \hbar \omega$ space, and we solve an intrinsic Hamiltonian that depends on the relative coordinate in a model space consisting of single-particle coordinates. We consider different interaction parameters $V_{0}$, and set the oscillator length of our basis equal to the scale $l$ of the Gaussian interaction. Note that the resulting ground state has an extension that, depending on the strength $V_{0}$ of the interaction, differs considerably from $l$. Indeed, approximating the interaction by a parabola at its minimum shows that the corresponding frequency is $\Omega / \omega=\sqrt{2 V_{0} /(\hbar \omega)}$. Again, we find that the ground-state wave function has a Gaussian shape in the direction of the center-of-mass coordinate $\left(x_{1}+x_{2}\right) / \sqrt{2}$. Figure 10 quantifies this statement. The circles show the relative error of the groundstate energy (obtained from comparing the result in a model space of $N=8$ oscillator shells with the result in $N=16$ oscillator shells) as the strength $V_{0}$ of the interaction is varied. The squares (diamonds) show to what extent the ground-state wave function factorizes in a model space consisting of $N=8(N=16)$ oscillator shells. The residual $1-s_{1}^{2}$ is clearly very small, and it decreases with increasing size of the model space. The triangles show the ground-state expectation value of the center-ofmass Hamiltonian (27), normalized by the corresponding spacing $\hbar \tilde{\omega}$. Thus, the center-of-mass wave function factorizes, and it is to a high accuracy a Gaussian. The result obtained for this simple Hamiltonian lends further support to the results obtained in the coupled-cluster approach. It thus seems that one might obtain a very accurate factorization in sufficiently large model spaces even without employing an $N \hbar \omega$ space. Again, this factorization is not surprising since the model space becomes more and more complete as it increases in size. The emergence of a Gaussian center-of-mass wave function, however, is remarkable and not yet understood.

The center-of-mass problem in coupled-cluster calculations has also recently been addressed by Roth and coworkers [48]. These authors consider a Hamiltonian of the form $H=H_{\text {in }}+\beta H_{\mathrm{cm}}(\omega)$, that is, the center-ofmass Hamiltonian (27) with a frequency fixed to that of the employed harmonic oscillator basis is added to the intrinsic Hamiltonian. This method, pioneered by Gloeckner and Lawson [49], aims at shifting spurious states up in the spectrum. Roth et al. found that the coupledcluster wave function for such a Hamiltonian does not factorize into a Gaussian with frequency $\omega$ for centerof-mass wave function and an intrinsic wave function. Two comments are in order. First, we believe that this approach misses the point as it requires perhaps more than the coupled-cluster approximation needs to deliver. For atomic nuclei, there is no center-of-mass interaction, and the requirement that the wave function factorizes in presence of a center-of-mass interaction is more than one needs. Some time ago, McGrory and Wildenthal also

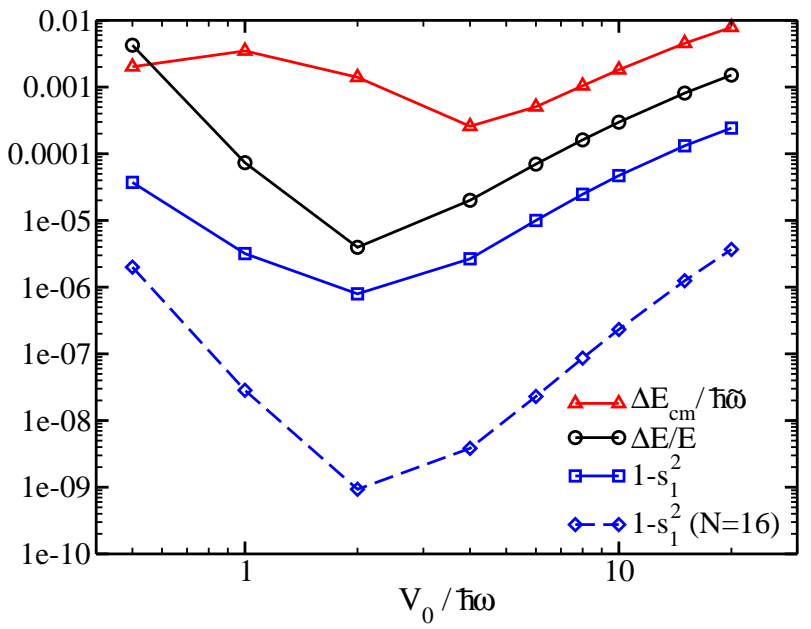

FIG. 10: (Color online)Results for the toy model (33) as a function of the strength of the interaction $V_{0}$. Circles: Estimate of relative error $\Delta E / E$ of the ground-state energy by comparing a calculation in $N=8$ oscillator shells with a calculation in $N=16$ shells. Squares and diamonds: Fraction $1-s_{1}^{2}$ of the ground state wave function that is not factorized in an intrinsic state and a center-of-mass state, obtained from the singular value decomposition (28) in a model space of $N=8$ and $N=16$ oscillator shells, respectively. Triangles: The ground-state expectation value $E_{\mathrm{cm}}(\tilde{\omega})$ of the harmonic oscillator center-of-mass Hamiltonian is much smaller than the energy $\hbar \omega$ of spurious center-of-mass excitations, indicating that the center-of-mass wave function is approximately a Gaussian.

pointed out that the method by Gloeckner and Lawson is not fully appropriate in truncated model spaces that are not complete $N \hbar \omega$ spaces [50]. For nuclei, one only needs that the wave function of the intrinsic Hamiltonian, when computed in a Hilbert space of $A$ coordinates, exhibits a factorization. Second, we caution that the conclusions by Roth and coworkers are based on results that are not fully converged with respect to the size of the model space (see Sect. VIII below).

The results of this section suggest the following procedure when dealing with model spaces that are not complete $N \hbar \omega$ spaces: (i) Compute the spectrum of the intrinsic Hamiltonian $H_{\text {in }}$ in as large a model space as conveniently possible. (ii) Check a posteriori whether the resulting center-of-mass wave function is a Gaussian by computing the expectation value $E \mathrm{~cm}$, and determine the corresponding frequency $\tilde{\omega}$. (iii) The ratio $E_{\mathrm{cm}} /(\hbar \tilde{\omega})$ serves as a conservative estimate for the quality of the factorization. (Strictly speaking, this ratio measures to what extent the center-of-mass wave function deviates from a Gaussian with frequency $\tilde{\omega}$.) 


\section{COUPLED-CLUSTER RESULTS FOR MEDIUM-MASS NUCLEI WITH CHIRAL INTERACTIONS}

In this section, we compute binding energies and radii of several doubly magic nuclei within the CCSD and the $\Lambda$-CCSD(T) approximation, and employ chiral NN interactions.

Our single-particle wave functions are eigenfunctions of the harmonic oscillator and characterized by the frequency $\omega$. Our model space consists of spherical oscillator wave functions with radial quantum number $n$ and angular momentum $l$, and we include single-particle states with $2 n+l \leq N$ and $l \leq 10$. Fully converged energies have to be independent of the parameters $N$ and $\omega$ of our single-particle basis. In practice, we cannot go to infinitely large spaces, but this is also not necessary for the description of low-energy properties of finite nuclei. Our basis needs to be complete in the following sense. It must be sufficiently extended in momentum space to resolve the cutoff $\Lambda_{\chi}$ of the employed interaction, and its extension in position space must be such that a nucleus of radius $R$ literally fits into the basis. Let us estimate the parameters required for a model space of oscillator functions.

An oscillator basis consisting of $N$ shells at a frequency $\omega$ resolves the high momentum cutoff $\lambda$ if the inequality

$$
N \hbar \omega \gtrsim \frac{\hbar^{2} \lambda^{2}}{m}
$$

is fulfilled. Likewise, the basis has to be sufficiently extended in position space to describe a nucleus with a radius $R$. For this, the inequality

$$
\hbar \omega \lesssim N \frac{\hbar^{2}}{m R^{2}}
$$

needs to be fulfilled. In other words, an oscillator basis with oscillator length $l_{\text {ho }}=\sqrt{\hbar /(m \omega)}$ exhibits the infrared cutoff $l_{\text {ho }}^{-1} / \sqrt{N}$ and the ultraviolet cutoff $l_{\text {ho }}^{-1} \sqrt{N}$. Thus, converged results require model space parameters $(N, \omega)$ that fulfill the inequalities (34) and (35), and the results will then be insensitive to the specific values of the parameters. Note that the simultaneous fulfillment of Eq. (35) and Eq. (34) requires $N \gtrsim \lambda R$. (Our calculations presented in this paper show that these approximate relations are reasonable estimates.) Note also that

$$
\hbar \omega \approx \frac{\hbar^{2} \lambda}{m R},
$$

for a minimum model space with $N \approx \lambda R$. Thus, the well-known estimate $\hbar \omega \approx 42 / A^{1 / 3} \mathrm{MeV}$ is only valid for small cutoffs $\lambda \approx k_{F}$ that are close to the Fermi momentum $k_{F}$. These considerations show that much is to be gained from low-momentum interactions, and - conversely - that "bare" chiral interactions with $\lambda=\Lambda_{\chi}$ require very large model spaces.
Let us consider the oscillator basis as an example. A model space of $N$ oscillator states contains about $\Omega \approx$ $N^{3} / 3$ single-particle states. We obtain converged results for ${ }^{16} \mathrm{O}$ and the interactions from chiral EFT in $N \approx 15$ shells. In this model space there are $\Omega ! /((\Omega-Z) ! Z !) \approx$ $10^{20}$ Slater determinants for the protons alone. Thus, the resulting model space for protons and neutrons is far out of reach from diagonalization methods.

We employ the chiral NN interaction at order $\mathrm{N}^{3} \mathrm{LO}$, i.e., the $\mathrm{NN}$ interaction is included up to the order $\left(Q / \Lambda_{\chi}\right)^{3}$, while $3 \mathrm{NF}$ at this order and interactions at higher order are neglected. We perform a spherical Hartree-Fock calculation and transform the Hamiltonian to the Hartree-Fock basis. The CCSD equations are then solved in the spherical Hartree-Fock basis, and the Hartree-Fock state is taken as the reference state for the coupled-cluster method. Note that the chiral $N^{3}$ LO interaction is quite "hard". Within the Hartree-Fock approximation one does not even obtain bound nuclei. Nevertheless, the solution of the non-linear coupled-cluster equations yields rather well-bound nuclei.

Fig. 11 shows the ground-state energy of ${ }^{4} \mathrm{He}$ in a model space of $N=18$ (19 major oscillator shells). In such a large model space, the results are virtually independent of the frequency $\omega$ over a wide range. The CCSD result for ${ }^{4} \mathrm{He}$ deviates from the virtually exact result from the solution of the Faddeev-Yakubowsky (FY) equations by about $6 \%$. This difference is due to the omission of three- and four-particle clusters. The more accurate $\Lambda$-CCSD(T) method includes three-body clusters approximately and overshoots the (FY) result by about $1.5 \%$. This overbinding is due to the non-variational character of the coupled-cluster method and is not really a concern due to the accuracy of the method. The experimental ground-state energy of ${ }^{4} \mathrm{He}$ is $E=-28.3 \mathrm{MeV}$, and the additional binding must be attributed to the $3 \mathrm{NFs}$ at order $\mathrm{N}^{3} \mathrm{LO}$; other high-order terms in the chiral Langrangian play only a very small role in ${ }^{4} \mathrm{He}$.

We also computed the radius of the alpha particle within the Hellmann-Feynman approach. The results shown in Fig. 12 exhibit a very weak dependence on the oscillator frequency, particularly within the $\Lambda$-CCSD $(\mathrm{T})$ approximation. Within this approach, the radius deviates by about $0.01 \mathrm{fm}$ from the NCSM results [51], and about $0.015 \mathrm{fm}$ from the virtually exact result from the hyperspherical harmonics method employed by the Pisa group [4]. We believe that the results presented for the alpha particle manifest the high degree of accuracy that the coupled-cluster approximation exhibits. Note that the alpha particle also exhibits the approximate factorization into a Gaussian center-of-mass wave function and an intrinsic wave function. Here, the frequency of the Gaussian is $\hbar \tilde{\omega} \approx 19 \mathrm{MeV}$ while the expectation value of the center-of-mass Hamiltonian is much smaller $E_{\mathrm{cm}} \approx 0.3 \mathrm{MeV}$.

We next turn to the calcium isotopes ${ }^{40} \mathrm{Ca}$ and ${ }^{48} \mathrm{Ca}$. Figure 13] shows the CCSD and $\Lambda$-CCSD $(\mathrm{T})$ ground state energy for ${ }^{40} \mathrm{Ca}$ as a function of the oscillator frequency 


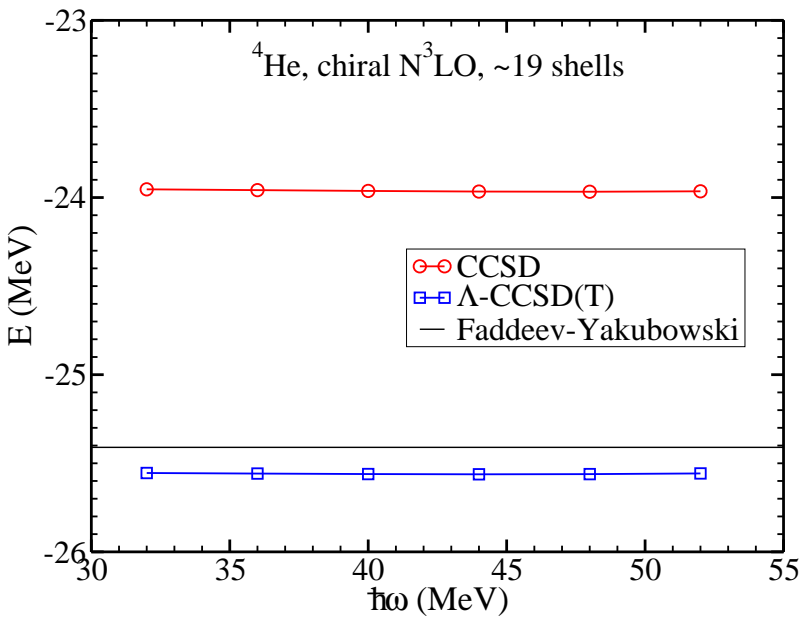

FIG. 11: (Color online) CCSD and $\Lambda$-CCSD $(\mathrm{T})$ ground state energy of ${ }^{4} \mathrm{He}$ using a chiral $\mathrm{NN}$ interaction at order $\mathrm{N}^{3} \mathrm{LO}$ in 19 major oscillator shells as a function of the oscillator spacing $\hbar \omega$, compared to virtually exact results from FaddeevYakubowsky calculations.

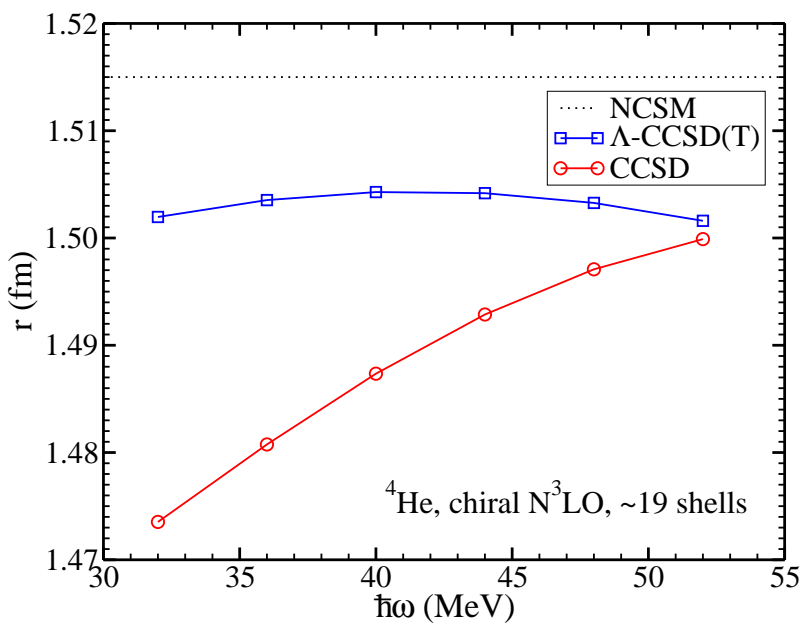

FIG. 12: (Color online) ${ }^{4} \mathrm{He}$ RMS radii calculated using the Hellmann-Feynman theorem within the CCSD and the $\Lambda$ $\operatorname{CCSD}(\mathrm{T})$ approximations using a chiral NN interaction at order $\mathrm{N}^{3} \mathrm{LO}$ with cutoff $500 \mathrm{MeVc}^{-1}$. Calculations employed 19 major oscillator shells, and the results are plotted as a function of the oscillator spacing $\hbar \omega$.

$\hbar \omega$ and the size of the model space $N$ (here the number of major shells is $N+1)$. For $\hbar \omega=32 \mathrm{MeV}$ and $N+1=15$ shells the $\Lambda$-CCSD(T) ground state energy is $-345.074 \mathrm{MeV}$. We also performed a calculation in $N+1=19$ shells at $\hbar \omega=32 \mathrm{MeV}$, yielding a $\Lambda-\operatorname{CCSD}(\mathrm{T})$ ground state energy of $-345.781 \mathrm{MeV}$. This shows that our results are converged within 1-2 $\mathrm{MeV}$ with respect to the size of the model space. Within this approximation, one overbinds ${ }^{40} \mathrm{Ca}$ by about $3 \mathrm{MeV}$. Compared to the previously published CCSD results, the triples corrections add more than $30 \mathrm{MeV}$ of binding.

Figure 14 shows the $\Lambda$-CCSD(T) results for ${ }^{48} \mathrm{Ca}$. The

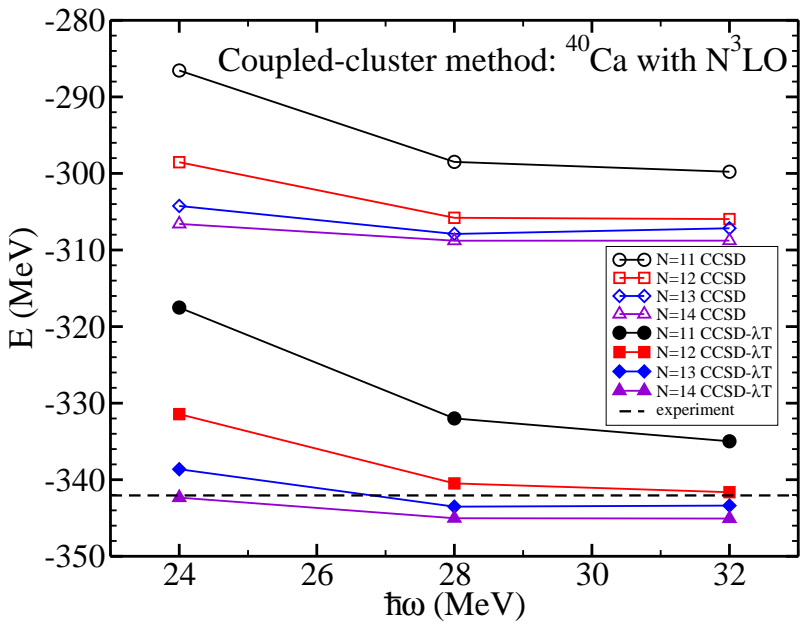

FIG. 13: (Color online) $\Lambda$-CCSD(T) and CCSD results for ${ }^{40} \mathrm{Ca}$ from the chiral NN interaction at order $\mathrm{N}^{3} \mathrm{LO}$ as a function of the oscillator spacing $\hbar \omega$ and the size of the model space.

results are converged to within about $2 \mathrm{MeV}$ in a model space of $N+1=19$ shells, and only exhibit a weak model-space dependence in the largest model space. The approximation with triples yields about an additional $40 \mathrm{MeV}$ of binding when compared to the CCSD results. Let us assume that the difference between our calculations and the experiment is mainly due to the omitted 3 NFs. This suggests that the three-nucleon forces (3NF) will exhibit an interesting isospin dependence. For ${ }^{40} \mathrm{Ca}$, only small (repulsive) contributions from the $3 \mathrm{NF}$ are expected, while about $20 \mathrm{MeV}$ of attraction is needed for ${ }^{48} \mathrm{Ca}$. A similar picture arises when comparing ${ }^{16} \mathrm{O}$ with ${ }^{22} \mathrm{O}$. For the former, about $0.41 \mathrm{MeV}$ per nucleon in binding are missing, while the latter lacks about $0.82 \mathrm{MeV}$ per nucleon in binding [27].

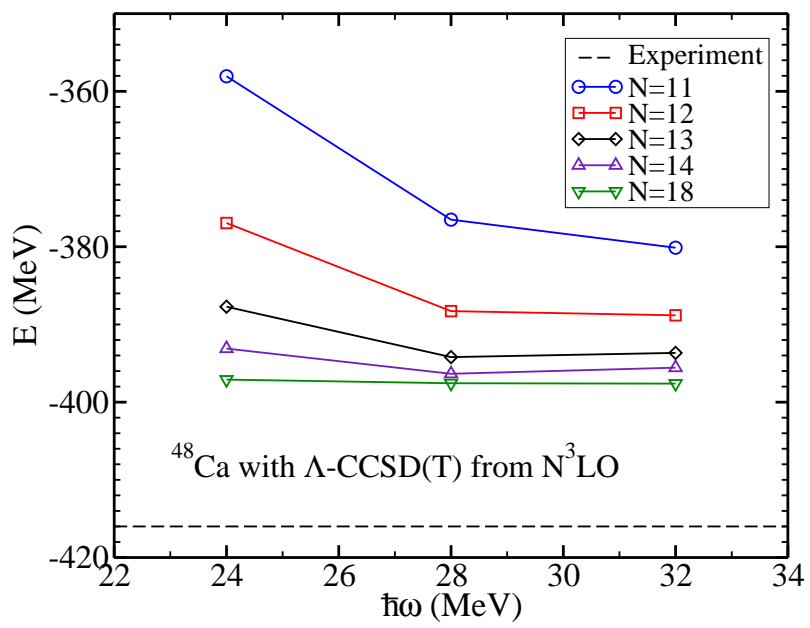

FIG. 14: (Color online) $\Lambda$-CCSD $(\mathrm{T})$ and CCSD results for ${ }^{48} \mathrm{Ca}$ from the chiral NN interaction at order $\mathrm{N}^{3} \mathrm{LO}$ as a function of the oscillator spacing $\hbar \omega$ and the size of the model space. 
At this point, it is interesting, to compare the energies from the Hartree-Fock calculation, the CCSD calculation, and the $\Lambda$-CCSD(T) approximation. This comparison will permit us to estimate the precision of the coupled-cluster method. Table @ shows that CCSD accounts for about $90 \%$ of the correlation energy, while the $\Lambda$-CCSD(T) approximation yields about $10 \%$. This hierarchy has been observed in quantum chemistry as well, see for example Ref. [33]. For the alpha particle we know that four-particle clusters account again for about $10 \%$ of the triples correction. Thus, it makes no sense to artificially increase the precision of our results by turning to even larger model spaces. The error estimates due to the finite model space are of the same order as the estimates due to omitted four-body clusters. Note that the results in Table \are also consistent with the size extensivity of the employed coupled-cluster methods, as the deviation $\Delta E$ from data is approximately linear in mass number, i.e., $\Delta E / A$ is approximately constant over a considerable range.

\begin{tabular}{|c|r|r|r|r|}
\multicolumn{2}{c}{ CCSD } & \multicolumn{2}{c}{$\mathrm{CCSD}(\mathrm{T})$} \\
\hline Nucleus & $E / A$ & $\Delta E / A$ & $E / A$ & $\Delta E / A$ \\
\hline${ }^{16} \mathrm{O}$ & -6.72 & 1.25 & -7.56 & 0.41 \\
\hline${ }^{40} \mathrm{Ca}$ & -7.72 & 0.84 & -8.63 & -0.08 \\
\hline${ }^{48} \mathrm{Ca}$ & -7.40 & 1.27 & -8.26 & 0.40 \\
\hline
\end{tabular}

TABLE I: Ground-state energies per nucleon $E / A$ and deviation $\Delta E / A$ from experiment for doubly magic nuclei within the CCSD and $\Lambda$-CCSD(T) approximations. All energies are in units of $\mathrm{MeV}$.

It is also very interesting to compare our results with the results [52] by Fujii et al. obtained within the Unitary Model Operator Approach 53]. For ${ }^{16} \mathrm{O}$, Fujii et al. report binding energies of $6.62 \mathrm{MeV}$ per nucleon and $7.47 \mathrm{MeV}$ per nucleon employing two-body clusters and three-body clusters, respectively. The close agreement between our results presented in Table @ and those of Ref. [52] demonstrate that different $a b$ initio methods are setting reliable benchmarks for increasingly heavier nuclei.

\section{SHELL EVOLUTION WITH CHIRAL NN INTERACTIONS}

In this section we investigate the evolution of the lowlying positive parity states $1 / 2^{+}, 3 / 2^{+}$, and $5 / 2^{+}$in the oxygen and fluorine isotope chains using the chiral nucleon-nucleon interaction at $\mathrm{N}^{3} \mathrm{LO}$. Our calculations do not include three-body forces, but in order to probe the effects of omitted short-ranged many-body forces we renormalize the "bare" chiral interaction using the similarity renormalization group method and study the resolution scale dependence on the calculated energies. The low-lying states $J^{\pi}=1 / 2^{+}, J^{\pi}=3 / 2^{+}$, and $J^{\pi}=5 / 2^{+}$ in oxygen and fluorine are usually interpreted as the $s_{1 / 2}$, $d_{3 / 2}$, and $d_{5 / 2}$ proton and neutron single-particle states in the closed-shell oxygen isotopes ${ }^{16} \mathrm{O},{ }^{22} \mathrm{O},{ }^{24} \mathrm{O}$, and ${ }^{28} \mathrm{O}$. Here, we define the single-particle energy of the state $J^{\pi}$ as the difference in binding energy between the $A \pm 1$ nucleus and the closed-shell nucleus $A$, i.e.,

$$
E_{\mathrm{s} p}\left(J^{\pi}\right)=E^{A \pm 1}\left(J^{\pi}\right)-E_{0}^{A}
$$

The evolution of nuclear shell structure for neutronrich isotopes is of great theoretical and experimental interest, see for example the recent review [54]. Some of the traditional magic numbers of the nuclear shell model might fade away as one moves away from the valley of beta stability, while new magic numbers emerge in neutron-rich nuclei. The evolution of shell structure in the isotopes of oxygen has recently received considerable experimental [55 58] and theoretical attention [27, 59 63. Considerable shell gaps have been observed in ${ }^{22} \mathrm{O}$ [55] and ${ }^{24} \mathrm{O}$ [56, 57], leading to the interpretation of new magic numbers at $(Z=8, N=14)$ and $(Z=8, N=16)$, respectively. Hoffman et al. 58] found that ${ }^{25} \mathrm{O}$ is a resonance and unstable towards one-neutron emission. The instability of ${ }^{25} \mathrm{O}$ is closely related to the location of the $d_{3 / 2}$ single-particle shell, and it is clear that the evolution of the $d_{3 / 2}$ shell will decide whether ${ }^{24} \mathrm{O}$ or ${ }^{28} \mathrm{O}$ is the most neutron-rich stable isotope of oxygen.

The underlying microscopic mechanisms for the evolution of nuclear shell structure is not yet entirely understood. One might expect that both three-nucleon forces and coupling with the scattering continuum will play significant roles in predicting the limits of nuclear stability and the shell evolution towards the drip line. Zuker 64 suggested that three-nucleon forces modify the monopole terms of microscopically derived shell-model interactions. Indeed, Otsuka et al. 62] found within the $s d$ shell model that 3 NFs will add repulsion between the $d_{5 / 2}$ and $d_{3 / 2}$ orbitals, thus making ${ }^{24} \mathrm{O}$ the heaviest bound isotope of oxygen. Coupled-cluster calculations of the isotopes $16,22,24,28 \mathrm{O}$ reveal that three-nucleon forces (or more complicated many-body forces) play a non-negligible role in the determination of the drip-line for oxygen isotopes.

In this section, we study the evolution of shell structure in the isotopes of oxygen and fluorine based on chiral NN interactions [13]. We will omit continuum effects and $3 \mathrm{NFs}$ from our study. Recall that chiral 3NFs consist of a long-ranged two-pion exchange, a mid-range one-pion exchange, and a contact term [65]. Variation of the cutoff will induce short-range $3 \mathrm{NF}$ s that are in their structure identical to the chiral $3 \mathrm{NF}$ [66, 67]. Thus, we will be able to probe the effect of short-ranged 3NFs a posteriori by cutoff variation. Continuum effects are, of course, expected to be important for nuclei close to the drip line [18, 68, 69]. However, it will turn out that most of the computed single-particle states are fairly well bound (likely due to the omission of $3 \mathrm{NFs}$ ). In this situation, the continuum plays a smaller role.

Figures [15] and [16] show the effective single-particle energies for the neutron and proton $s_{1 / 2}, d_{3 / 2}$, and $d_{5 / 2}$ 
states in ${ }^{16} \mathrm{O},{ }^{22} \mathrm{O},{ }^{24} \mathrm{O}$, and ${ }^{28} \mathrm{O}$. The calculations employed a model space consisting of $N+1=15$ oscillator shells at a fixed oscillator frequency $\hbar \omega=28 \mathrm{MeV}$. The proton $s_{1 / 2}, d_{3 / 2}$, and the $d_{5 / 2}$ single-particle states were computed within the PA-EOM-CCSD approach. The neutron single-particle states resulted from either the PR-EOM-CCSD or the PA-EOM-CCSD method (See subsection IIB for more details), depending on whether the considered single-particle state is a hole or a particle state of an isotope of oxygen with a closed subshell.

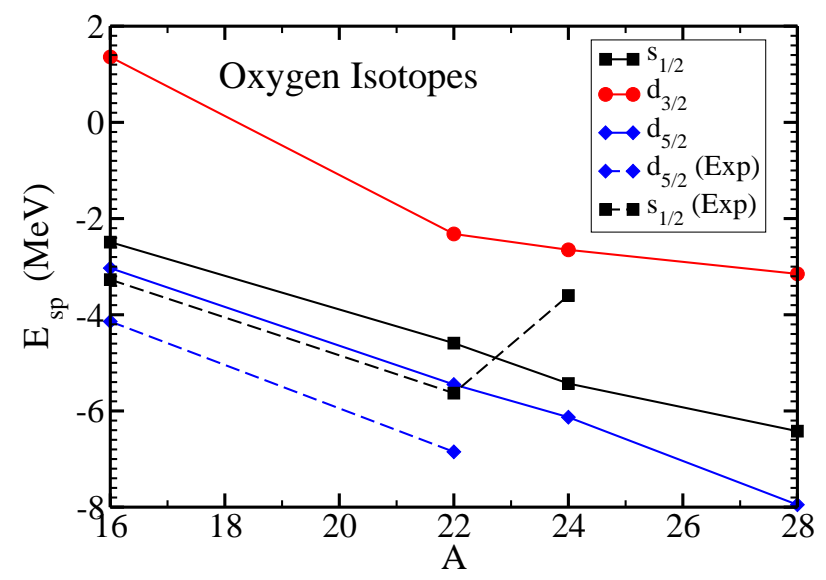

FIG. 15: (Color online) Single-particle energies (Eq. (37)) of the $s_{1 / 2}, d_{3 / 2}$, and the $d_{5 / 2}$ single-particle states in the oxygen isotopes using the "bare" chiral NN interaction. The calculations employ $N=14$ shells for fixed $\hbar \omega=28 \mathrm{MeV}$. The $d_{5 / 2}$ and $s_{1 / 2}$ experimental single-particle energies are shown as dashed lines.

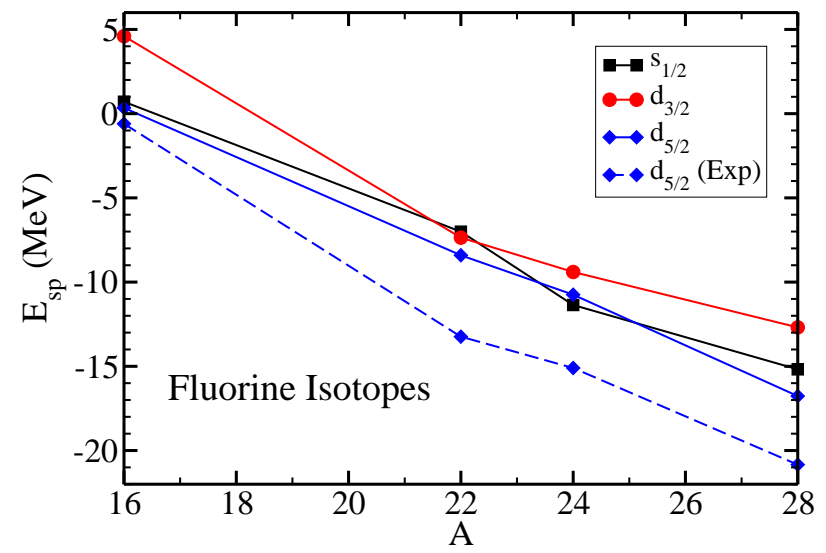

FIG. 16: (Color online) Single-particle energies (Eq. (37)) of the $s_{1 / 2}, d_{3 / 2}$, and the $d_{5 / 2}$ single-particle states in the fluorine isotopes using the "bare" chiral NN interaction. The calculations employ $N=14$ shells for fixed $\hbar \omega=28 \mathrm{MeV}$. The experimental $d_{5 / 2}$ single-particle energiy is shown as a dashed line.

Several interesting features can be extracted from the results. The computed neutron single-particle energies (shown in Fig. 15) do not reproduce the experimentally observed shell gaps in ${ }^{22} \mathrm{O}$ and ${ }^{24} \mathrm{O}$. Furthermore, the chiral NN interactions incorrectly yield a bound ${ }^{25} \mathrm{O}[58]$. This behavior has also been seen in shell-model calculations of the oxygen isotopes using microscopically derived shell-model interactions (see for example Ref. [62]). For the fluorine isotopes (shown in Fig. 16), the $d_{5 / 2}$ state follows the experimental trend but lacks binding. In ${ }^{25} \mathrm{~F}$ our calculations yield an inversion of the $s_{1 / 2}$ and $d_{5 / 2}$ states, giving a $1 / 2^{+}$state as the ground state of ${ }^{25} \mathrm{~F}$. In order to gauge the effects of omitted short-range $3 \mathrm{NFs}$, we vary the resolution scale $\lambda$ by a similarity renormalization group (SRG) transformation [19].

Figure 17] shows the dependence of the neutron $d_{3 / 2}$ single-particle state in ${ }^{24} \mathrm{O}$ as a function of the SRG cutoff $\lambda$. In these calculations we used $N+1=15$ oscillator shells with a fixed oscillator frequency of $\hbar \omega=26 \mathrm{MeV}$.

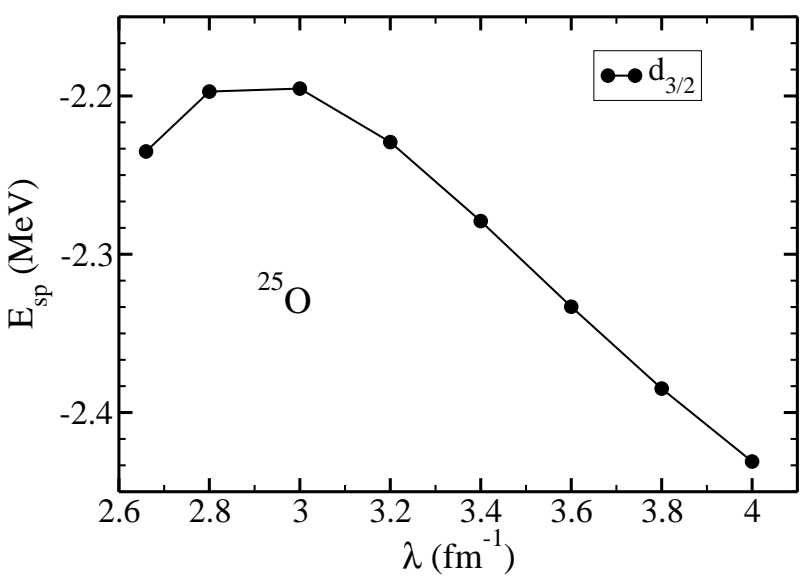

FIG. 17: (Color online) Single-particle Energy (Eq. (37)) of the neutron $d_{3 / 2}$ state in ${ }^{24} \mathrm{O}$ as a function of the resolution scale $\lambda$ of the SRG evolved chiral NN interaction. The calculations employ $N=14$ shells at fixed $\hbar \omega=26 \mathrm{MeV}$.

The $d_{3 / 2}$ single-particle state in ${ }^{24} \mathrm{O}$ depends weakly on the cutoff and stays bound for all choices of the cutoff $\lambda$. This suggests that long-ranged 3NFs are needed to yield an unbound state and to place the drip line at ${ }^{24} \mathrm{O}$. Furthermore, the effect of the scattering continuum is expected to add some additional binding to this state (see Ref. [18]). Thus, a realistic description of ${ }^{25} \mathrm{O}$ will result from a fine interplay between the scattering continuum and 3NFs. Figure 18 shows the cutoff dependence of the CCSD and $\Lambda$-CCSD(T) ground-state energies for ${ }^{24} \mathrm{O}$ (using the same model space and oscillator frequency as in Fig. 177). Note that there is no cutoff $\lambda$ that simultaneously reproduces the experimental binding energy of ${ }^{24} \mathrm{O}$ and the resonance energy of ${ }^{25} \mathrm{O}$ ground state. Figure 19 shows that proton $s_{1 / 2}, d_{3 / 2}$, and $d_{5 / 2}$ single-particle states in ${ }^{24} \mathrm{O}$ exhibit a considerable dependence on the cutoff $\lambda$. No single cutoff reproduces simultaneously the ground-state binding energies of ${ }^{24} \mathrm{O},{ }^{25} \mathrm{O}$, and ${ }^{25} \mathrm{~F}$, again pointing to the importance of the omitted $3 \mathrm{NFs}$ and to subtleties in its isospin dependence.

In conclusion, chiral NN interactions alone do not reproduce the evolution of single-particle energies within 


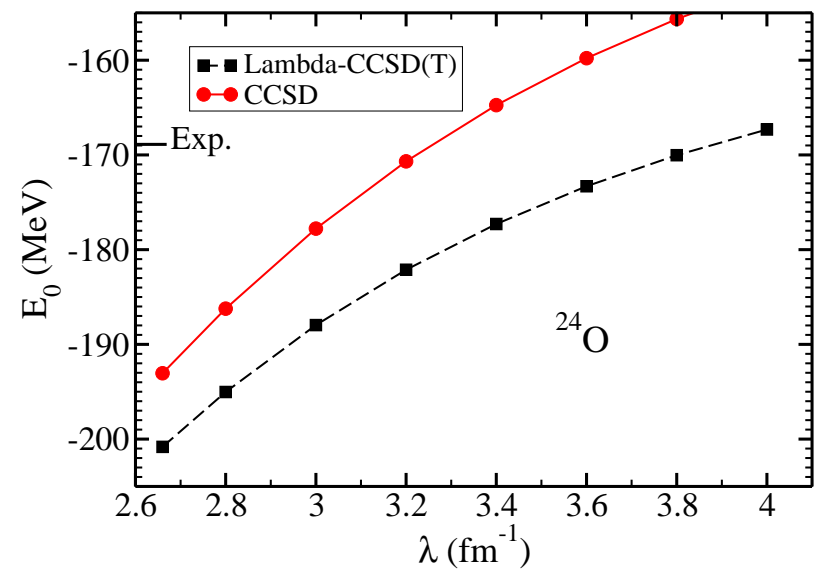

FIG. 18: (Color online) Ground-state energy of ${ }^{24} \mathrm{O}$ within the CCSD (circles) and the $\Lambda$-CCSD $(\mathrm{T})$ approximation (squares) as a function of the resolution scale $\lambda$ of the SRG evolved chiral NN interaction. The calculations employ in $N+1=15$ shells at fixed $\hbar \omega=26 \mathrm{MeV}$.

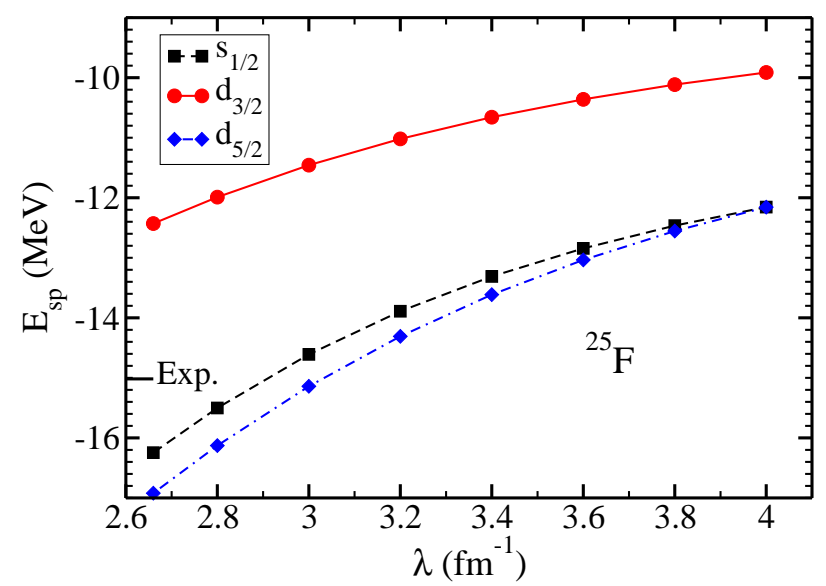

FIG. 19: (Color online) Single-particle energies (Eq. (37)) of the proton $s_{1 / 2}, d_{3 / 2}$, and $d_{5 / 2}$ states in ${ }^{24} \mathrm{O}$ as a function of the resolution scale $\lambda$ of the SRG evolved chiral NN interaction. The calculations employ $N+1=15$ shells at fixed $\hbar \omega=26 \mathrm{MeV}$.

the $s d$ shell. Close to ${ }^{16} \mathrm{O}$, the single-particle states are in semi-quantitative agreement with data, but missing $3 \mathrm{NFs}$ become increasingly more important for neutronrich isotopes of oxygen. It is expected, that a realistic description of single-particle states and the evolution of shell structure will require a theory which allows for a consistent and systematic inclusion of many-body correlations, $3 \mathrm{NFs}$, and coupling to the scattering continuum. The beauty of coupled-cluster theory is that it allows for inclusion of all these ingredients in a simple and transparent way, and we aim to investigate and predict properties of nuclei from the valley of stability to the very limits of matter taking all these ingredients into account in the near future.

\section{RESOLUTION-SCALE DEPENDENCE FOR ${ }^{40}$ CA AND POWER COUNTING}

In this section we study the ground-state properties of ${ }^{40} \mathrm{Ca}$ using renormalized nucleon-nucleon interactions derived with the SRG method [19]. The SRG method drives the Hamiltonian to a band diagonal form, and therefore decouples low-momentum degrees of freedom from high-momentum degrees of freedom. The cutoff parameter $\lambda$ determines the decoupling and sets the resolution scale or energy scale with which we can probe the structure of a particular nucleus. However, this procedure induces three-body forces and forces of higher rank. At the two-body level, these interactions typically overbind medium-mass nuclei considerably, and show a strong dependence on the cutoff $\lambda$. The dependence on the cutoff $\lambda$ on calculated observables gives an indication of the missing physics and on the rol e of many-body forces not included in the calculation; only the sum of all forces induced by the renormalization is truly independent of the scale of resolution or the cutoff.

For the ground-state calculations of ${ }^{40} \mathrm{Ca}$, we use SRG interactions at the resolution scales $\lambda=2.5,2.2,1.9 \mathrm{fm}^{-1}$, and compute the binding energy within the CCSD approximation. We solve the CCSD equations in a spherical harmonic oscillator basis. The SRG interactions are soft and the corrections due to triples clusters are found to be rather small. For ${ }^{40} \mathrm{Ca}$ and a low-momentum interaction $V_{\text {low } k}$ with cutoff $\lambda=1.9 \mathrm{fm}^{-1}$, for instance, the CCSD binding energy is $E / A=12.28 \mathrm{MeV}$ and the CCSD(T) approximations yields an additional $0.29 \mathrm{MeV}$ per nucleon [70]. Therefore, we limit the computations for the SRG interactions to the CCSD approximation. Our focus is on the saturation and convergence properties of the SRG interactions in medium-mass nuclei and not on precision results.

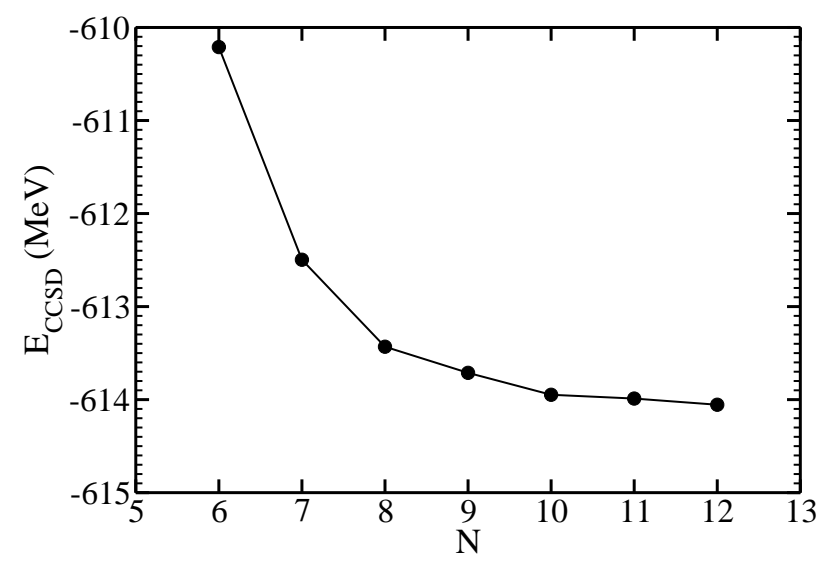

FIG. 20: (Color online) CCSD ground-state calculation of ${ }^{40} \mathrm{Ca}$ for increasing number of oscillator shells, $N=2 n+l$, at fixed $\hbar \omega=26 \mathrm{MeV}$, using SRG evolved chiral NN interaction at resolution scale $\lambda=1.9 \mathrm{fm}^{-1}$.

Figures 20, 21, and 22] show the convergence of the ground-state energy of ${ }^{40} \mathrm{Ca}$ using SRG evolved interac- 


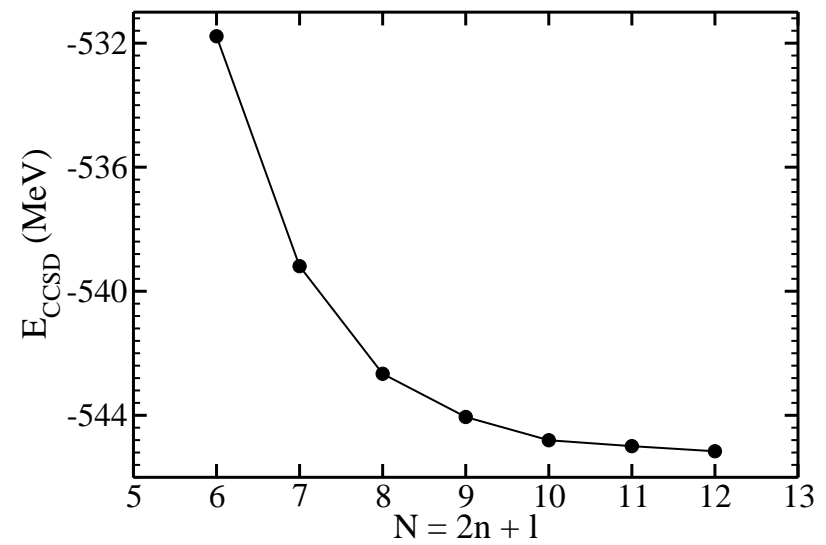

FIG. 21: (Color online) Same caption as in Fig. 20 except that resolution scale is $\lambda=2.2 \mathrm{fm}^{-1}$.

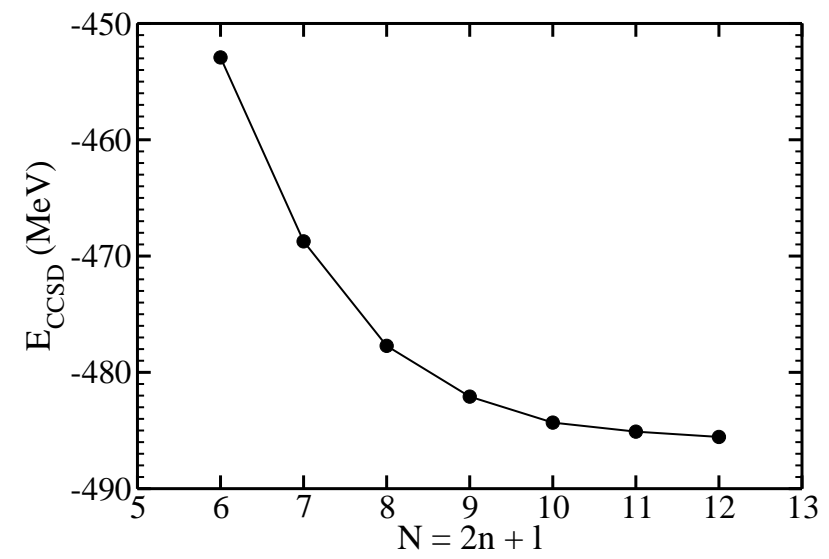

FIG. 22: (Color online) Same caption as in Fig. 20 except that resolution scale is $\lambda=2.5 \mathrm{fm}^{-1}$.

tions with resolution scales $\lambda=1.9,2.2,2.5 \mathrm{fm}^{-1}$, respectively. The ground-state energies computed for the various cutoffs are well converged in model space sizes comprising up to 13 major oscillator shells. As the cutoff $\lambda$ is lowered, these SRG interactions become increasingly soft, and we observe a faster convergence with increasing basis size. The computed ground-state energies show that the SRG NN interactions alone overbind considerably with respect to experiment. The results also exhibit a strong dependence on the resolution scale $\lambda$, and the overbinding with respect to the experimental binding energy of ${ }^{40} \mathrm{Ca}$, which is approximately $342 \mathrm{MeV}$, increases with decreasing momentum scale. This strong resolution dependence is, of course, due to our omission of threenucleon forces 3NFs (and more complicated many-body forces) and higher-order terms [66, 67]. Likewise, with increasingly softer NN interactions, the proper reproduction of saturation properties must come from the repulsive character of the $3 \mathrm{NF} 62$.

Table II shows the binding energy per particle $E / A$, interaction energy per particle $V / A$, the momentum expectation value $Q$, and the difference in binding energy per particle compared to experiment $\Delta E / A$ for ${ }^{40} \mathrm{Ca}$ us- ing the different SRG interactions. To examine the power

\begin{tabular}{|c|r|r|r|r|c|}
\hline$\lambda$ & $E / A$ & $V / A$ & $Q$ & $\Delta E / A$ & $\frac{\Delta E}{V} \mid /\left(\frac{Q}{\lambda}\right)^{3}$ \\
\hline 1.9 & -15.35 & -47.59 & 1.25 & -6.80 & 0.50 \\
2.2 & -13.63 & -44.84 & 1.23 & -5.08 & 0.62 \\
2.5 & -12.14 & -42.39 & 1.21 & -3.59 & 0.77 \\
\hline
\end{tabular}

TABLE II: CCSD results for ${ }^{40} \mathrm{Ca}$ with SRG interactions evolved from the chiral $\mathrm{N}^{3} \mathrm{LO}$ nucleon-nucleon interaction to the momentum scale $\lambda$. The binding energy per nucleon, and interaction energy per nucleon are denoted as $E / A$ and $V / A$, respectively. $Q$ denotes the expectation value of the momentum probed in this nucleus, while $\Delta E$ denotes the difference to the experimental binding energy. Energies are in units of $\mathrm{MeV}$ and momenta in units of $\mathrm{fm}^{-1}$.

counting, we compute the expectation value of the interaction energy via the Hellmann-Feynman theorem, and also deduce the average momentum $Q$ from the expectation value of the kinetic energy. The results are given in Table II. The CCSD results are very well converged for momentum scales $\lambda=1.9,2.2,2.5 \mathrm{fm}^{-1}$. As already pointed out, our results are far from complete. Due to the restriction to NN forces, we are missing contributions of $3 \mathrm{NFs}$ at order $\mathrm{N}^{3} \mathrm{LO}$, and $\mathrm{NN}, 3 \mathrm{NF}$, and $4 \mathrm{NF}$ forces from higher order. The missing contributions from clusters due to the CCSD approximation are much smaller than the contributions related to neglected terms of the interaction. Chiral effective field theory puts the contributions of $3 \mathrm{NFs}$ at order $(Q / \lambda)^{3}$. The SRG transformation shifts high-momentum NN contributions to forces of higher rank but is not expected to destroy the power counting. Are our results consistent with this expectation? To answer this question, we compute the ratio $|\Delta E / V| /(\lambda / Q))^{3}$ for the ${ }^{40} \mathrm{Ca}$ nucleus and present the result in Table II. The ratios are of natural size and suggest that the power counting is not violated in mediummass nuclei.

Let us discuss the implications of this finding. From a theoretical point of view, it is reassuring and satisfying that the power-counting arguments seem applicable in medium-mass nuclei. From a practical point of view, this raises the question about the order necessary to achieve a desired precision. Low-momentum and SRG versions of $\mathrm{NN}$ interactions are quite popular since they facilitate considerably the solution of the nuclear manybody problem. However, our results also suggest that the momentum expansion in $Q / \lambda$ has to be driven considerably beyond the present order $(Q / \lambda)^{3}$ in order to obtain a small error estimate due to missing contributions from the nuclear interactions. Recall that the $4 \mathrm{NF}$ enters at order $\mathrm{N}^{3} \mathrm{LO}$. Excluding cancellation among $4 \mathrm{NFs}$ (which might perhaps be achieved through some finetuning of low-energy constants), the technical advantage of soft NN interactions might interaction wise be offset due to the technical difficulties that come with the inclusion of forces of increasingly higher rank. This potential problem has not been noticed in light nuclei. In light nuclei, the contributions of $3 \mathrm{NFs}$ are reduced at rather 
small cutoffs due to fortuitous cancellations that are not expected from the power counting. Here, the lower cutoff benefits the handling of the NN force and it reduces the contributions of 3NFs. In light systems, there also seems to be only very little need for forces of rank higher than three. Table $\Pi$ hints that this might be different in ${ }^{40} \mathrm{Ca}$. Here, and in agreement with the power counting, the contributions of 3NFs increase with the decreasing momentum scale of the underlying interaction.

The results reported in this section suggest that there are at least two possible routes to ab-initio calculations in medium-mass nuclei. First, one can employ a low resolution scale $\lambda$ which facilitates the solution of the nuclear many-body problem. The price tag to be paid might consist of a slower convergence with respect to the order $k_{F} / \lambda$, i.e., the evolution of four-body forces might be needed. Second, one can employ a high cut-off scale $\Lambda_{\chi}$ and work on the technical difficulties related to the solution of the nuclear many-body problem. In this case, the full $\mathrm{N}^{3} \mathrm{LO}$ interaction including $3 \mathrm{NF}$ forces might already suffice to achieve accuracies of the order of $0.3 \mathrm{MeV}$ per nucleon, and fine-tuning of poorly constrained coefficients of the $3 \mathrm{NF}$ might further increase the accuracy. In following the second path, it might be attractive to employ other techniques to tame the "hard" NN interaction. In the next section, we therefore explore the convergence properties of the $G$-matrix computed from the chiral NN interaction at order $\mathrm{N}^{3} \mathrm{LO}$.

\section{CONVERGENCE PROPERTIES OF THE $G$-MATRIX}

In this section we calculate CCSD ground states of ${ }^{4} \mathrm{He}$ and ${ }^{16} \mathrm{O}$ using the in-medium $G$-matrix [22]. The $G$-matrix is an effective in-medium interaction that is computed starting from the bare nucleon-nucleon (NN) interaction using a Green's function approach with unperturbed propagators 23. It depends therefore on the starting energy $\bar{\omega}$. The starting energy defines the energy of the incoming and outgoing single-particle states, and is normally set equal to the sum of the unperturbed energies of the interacting single-particle states. The $G$-matrix is defined in a model space $P$ by summing ladder diagrams to infinite order, where the intermediate particle states are defined in the $Q$-space. Each ladder diagram scatters two particles from the $P$-space to the complement $Q$-space and back to the $P$-space. The number of interaction vertices in a ladder diagram gives the number of times the particles rescatter within the $Q$-space. It is clear that the $G$-matrix depends inherently on the model space and the interaction. The purpose of the $G$-matrix is to tame the hard core of the "bare" interaction making it suitable for many-body perturbation theory. In this section we investigate how the $G$-matrix behaves in $a b$-initio coupled-cluster ground-state calculations of ${ }^{4} \mathrm{He}$ and ${ }^{16} \mathrm{O}$ as the model space $P$ increases and the starting energy is varied over a wide range.
In constructing the $G$-matrix, we start from the "bare" $\mathrm{N}^{3} \mathrm{LO}$ interaction $\left(\Lambda_{\chi}=500 \mathrm{MeV}^{-1}\right)$ by Entem and Machleidt [13, 15] and a spherical harmonic oscillator basis. In Figs. 23 and 24, we show the CCSD results for ${ }^{4} \mathrm{He}$ and ${ }^{16} \mathrm{O}$, respectively, starting from the calculated $G$-matrix. In the calculations we varied the starting energy $\bar{\omega}$ from $-140 \mathrm{MeV}$ to $-5 \mathrm{MeV}$ and studied the effect of this variation on the CCSD ground state energy. We increased the model space from 10 to 14 major oscillator shells while the oscillator frequency was held fixed at $\hbar \omega=20 \mathrm{MeV}$. The figures show that the dependence on the starting energy is reduced as we increase the model space. In the largest model space considered here, the starting-energy dependence is very mild. As $\bar{\omega}$ is varied over a range of $135 \mathrm{MeV}$, the binding energy of ${ }^{4} \mathrm{He}$ changes only by about $0.25 \mathrm{MeV}$ and of ${ }^{16} \mathrm{O}$ by about $5 \mathrm{MeV}$. Furthermore, we see that at a fixed starting energy, the binding energy converges rather slowly with increasing size of the model space. As the model space $P$ increases the complement space $Q$ in which the particles scatter is reduced, and the $G$-matrix should therefore converge to the underlying "bare" nucleon-nucleon interaction in an infinite model space. This expectation is compatible with our CCSD results for the groundstate energies of ${ }^{4} \mathrm{He}$ (about $-24 \mathrm{MeV}$ ) and ${ }^{16} \mathrm{O}$ (about $-108 \mathrm{MeV}$ ) using the "bare" chiral NN interaction (see, e.g., Figs. 1 and 22). Figures 23 and 24 show that the ground-state energy of the $G$-matrix converges from below as the model space is inceased in size, and that the result of the "bare" interaction is approached in very large model spaces.

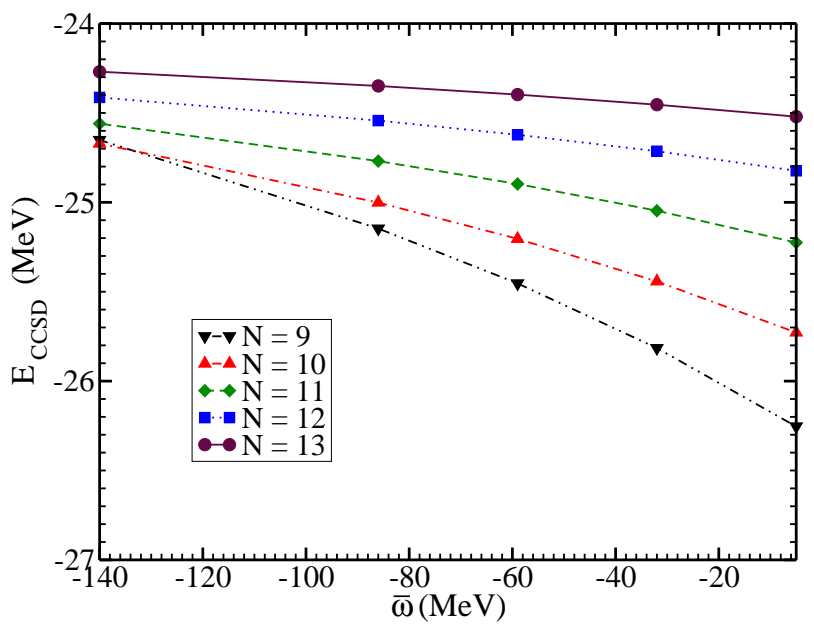

FIG. 23: (Color online) CCSD binding energy for ${ }^{4} \mathrm{He}$ using a $G$-matrix and starting energies $\bar{\omega}$ in the range from -140 $\mathrm{MeV}$ to $-5 \mathrm{MeV}$. The oscillator spacing is held fixed at $\hbar \omega=$ $20 \mathrm{MeV}$.

In conclusion, we observe slow convergence for the CCSD ground-state energy with increasing model space with the $G$-matrix defined from the "bare" $\mathrm{N}^{3} \mathrm{LO}$ interaction. The dependence on the starting energy disappears in sufficiently large model spaces, and the coupled-cluster 


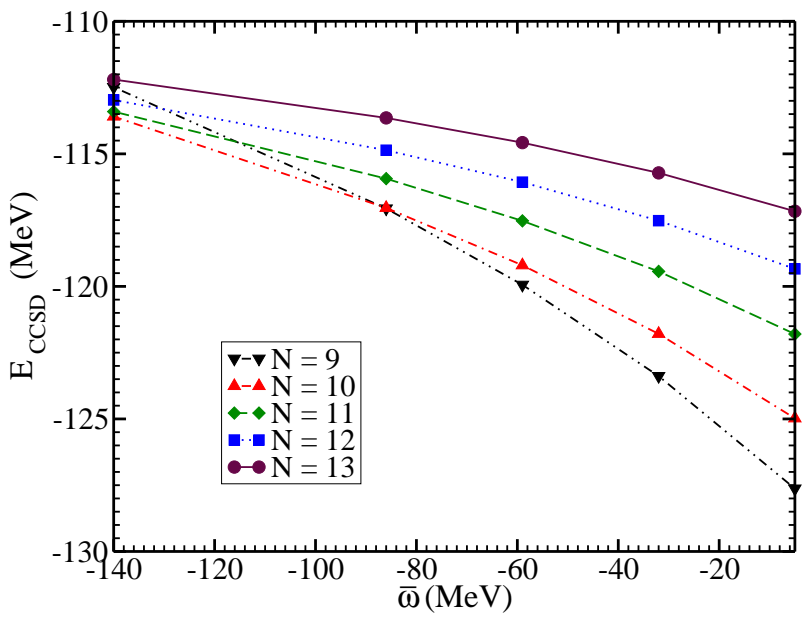

FIG. 24: (Color online) CCSD binding energy for ${ }^{16} \mathrm{O}$ using a $G$-matrix and starting energies $\bar{\omega}$ in the range from -140 $\mathrm{MeV}$ to $-5 \mathrm{MeV}$. The oscillator spacing is held fixed at $\hbar \omega=$ $20 \mathrm{MeV}$.

results with the $G$-matrix converge towards coupledcluster results for the free nucleon-nucleon interaction. In the case of the $\mathrm{N}^{3} \mathrm{LO}$ interaction, it is clearly better to start from the free interaction. It remains to be seen in the case of harder interactions, like the Argonne V18 interaction, whether convergence is better using the in-medium $G$-matrix as a starting point for $a b$ initio coupled-cluster calculations.

Let us briefly contrast the $G$ matrix to low-momentum $\mathrm{NN}$ interactions à la $V_{\text {low } k}$ or from the similarity renormalization group. The latter two preserve phase shifts from NN scattering and bound-state properties up to the chosen momentum cutoff. They soften the NN interaction and - when not augmented by the induced $3 \mathrm{NF}-$ tend to considerably overbind heavier nuclei at lower cutoffs around $\lambda \approx 2 \mathrm{fm}^{-1}$ or so. In a finite model space, the $G$-matrix always exhibits a dependence on the starting energy, it converges slowly to the "bare" interaction, and it somewhat overbinds the nuclear many-body system compared to the "bare" interactions that is used in its construction. The authors speculate that this last point might seem attractive with a view on practical applications, as "bare" nucleon-nucleon interaction models such as the Argonne interaction, the CD-Bonn interaction and the chiral interaction [13] somewhat underbind nuclei. In other words, the slight overbinding of the $G$-matrix often results into very reasonable energies for heavier nuclei, and the $G$-matrix simply works very well in practical applications.

\section{CONVERGENCE PROPERTIES OF $\mathrm{V}_{\mathrm{UCOM}}$}

Let us also consider the saturation and convergence properties of the $\mathrm{V}_{\mathrm{UCOM}}$ nucleon-nucleon interaction [24]. The interaction $\mathrm{V}_{\mathrm{UCOM}}$ is obtained from a uni- tary correlation operator method (UCOM) that softens the short-range repulsion of the initial "bare" interaction by a similarity transformation with a unitary operator, which is explicitly designed to remove the hard-core and the short-ranged tensor components of phenomenological $\mathrm{NN}$ interaction. In this section we consider the UCOM interaction obtained from the "bare" Argonne V18 interaction.

The $\mathrm{V}_{\mathrm{UCOM}}$ interaction was initially applied in meanfield methods. These applications were very successful. Very reasonable binding energies and saturation properties could already be obtained within the Hartree-Fock approximation. Only later did this interaction see applications that included the inclusion of many-body correlations within the RPA, many-body perturbation theory [71], the coupled-cluster method [72], and within the no-core shell model 73]. (For a recent review, we refer the reader to Ref. [74].) Those later applications suggest that the $\mathrm{V}_{\mathrm{UCOM}}$ interaction is soft yet with appealing saturation properties, and without the need for sizeable 3NFs. This situation seems surprising. On the one hand, the $\mathrm{V}_{\mathrm{UCOM}}$ interaction is similar in its technical construction to the SRG interactions [75]. On the other hand, the $\mathrm{V}_{\mathrm{UCOM}}$ interaction seems to differ significantly in its saturation property from the SRG nucleon-nucleon interactions which tend to overbind nuclei at low momentum cutoffs. We address this puzzle in what follows, and perform structure calculations with the UCOM interaction in large model spaces and for various oscillator frequencies.

We employ the $\mathrm{V}_{\mathrm{UCOM}}$ interaction and compute the ground states of ${ }^{16} \mathrm{O}$ and ${ }^{40} \mathrm{Ca}$ within the CCSD approximation. Our calculations employ very large model spaces and a considerable range of oscillator frequencies, and thereby differ from the previous studies $71-73]$; see also Ref. 25]. Figures 25] and 26 show the ground-state energy of ${ }^{16} \mathrm{O}$ and ${ }^{40} \mathrm{Ca}$, respectively, as a function of the employed oscillator frequency $\omega$ for different sizes of the model space $(N+1$ denotes the number of oscillator shells.). The convergence with respect to increasing size of the model space is slow, and we are unable to achieve convergence in up to 14 major oscillator shells. The results obtained in the largest model spaces also show that the $\mathrm{V}_{\mathrm{UCOM}}$ interaction considerably overbinds ${ }^{40} \mathrm{Ca}$. Within the CCSD approximation, we find a ground-state energy of about $-133 \mathrm{MeV}$ for ${ }^{16} \mathrm{O}$ and of about -400 $\mathrm{MeV}$ for ${ }^{40} \mathrm{Ca}$. The inclusion of triples corrections within the $\Lambda$ - $\operatorname{CCSD}(\mathrm{T})$ approximation yields a ground-state energy of $-140.99 \mathrm{MeV}$ for ${ }^{16} \mathrm{O}$ in 13 oscillator shells and an oscillator frequency $\hbar \omega=42 \mathrm{MeV}$.

We checked our results as follows. First, our CCSD results agree with those reported for ${ }^{4} \mathrm{He}$ in Ref. 72] within about $50 \mathrm{keV}$ in model spaces of up to eight oscillator shells. Second, we also computed the ground-state energy of ${ }^{40} \mathrm{Ca}$ in third order many-body perturbation theory. The relevant diagrams which are included can be found in Ref. 37]. Our results are shown in Fig. 27] as function of the oscillator energy $\hbar \omega$ and the number 


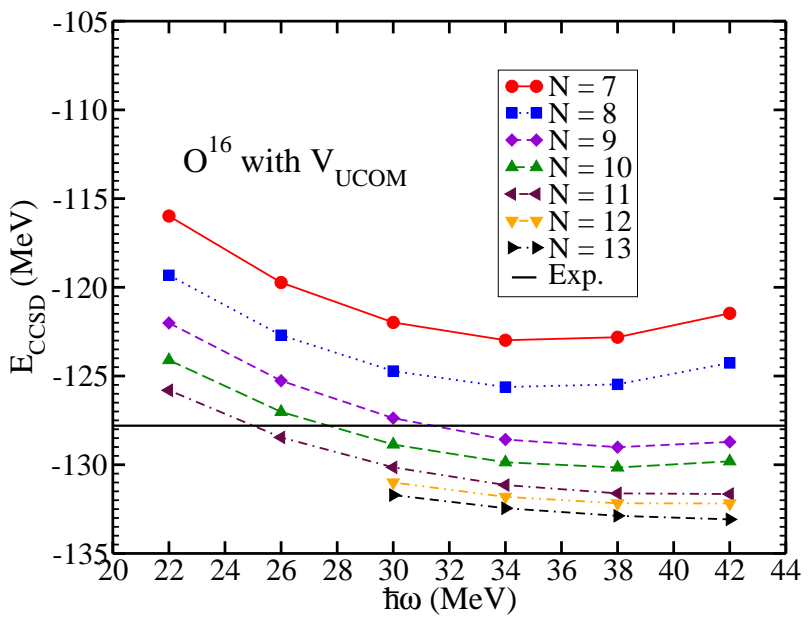

FIG. 25: (Color online) CCSD ground state energy of ${ }^{16} \mathrm{O}$ with $V_{\mathrm{UCO}}$ as a function of the oscillator spacing $\hbar \omega$ and the size of the model space $N$. The experimental groundstate energy is given by the solid line.

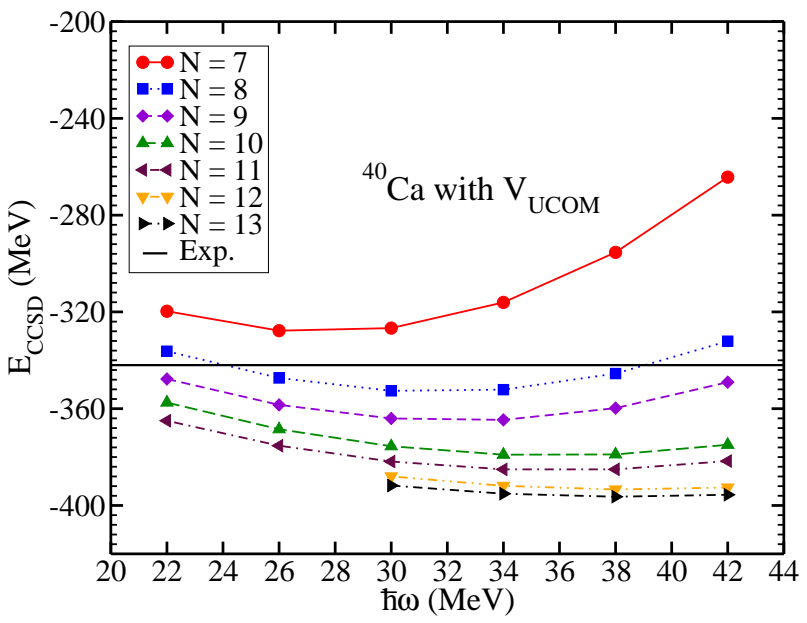

FIG. 26: (Color online) CCSD ground-state energy of ${ }^{40} \mathrm{Ca}$ using $V_{\mathrm{UCOM}}$ as a function of the oscillator spacing $\hbar \omega$ and the size of the model space $N$. The experimental ground state energy is given by the solid line.

of shells $N$. The results from many body perturbation theory confirm the trend seen in the coupled-cluster calculations, i.e., they exhibit a rather slow convergence in terms of the number of shells $N$ and overbind the nucleus ${ }^{40} \mathrm{Ca}$. Reference [71] also solved the UCOM potential within many-body perturbation theory, albeit for oscillator frequencies below $\hbar \omega \approx 20 \mathrm{MeV}$ or so. In this regime, the binding energy is closer to the experimental value as the small oscillator frequency acts as a cutoff in momentum space.

We speculate that the $\mathrm{V}_{\mathrm{UCOM}}$ interaction still exhibits relatively long tails in momentum space, which prevent a decoupling of low- and high-momentum modes, or that peculiarities of the corresponding cutoff yield a slow convergence of the UCOM interaction in an oscillator basis.

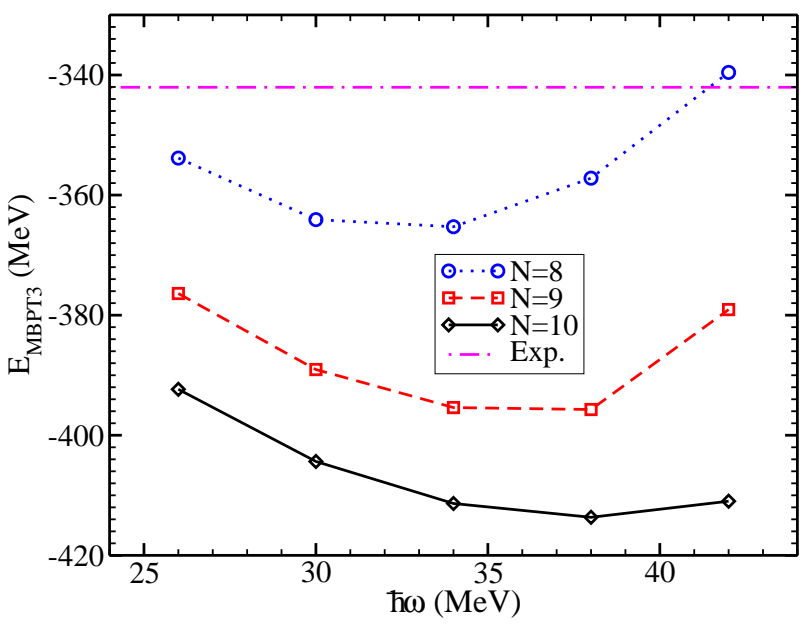

FIG. 27: (Color online) Ground-state energy for ${ }^{40} \mathrm{Ca}$ using many-body perturbation theory to third order in the interaction $V_{\mathrm{UCOM}}$. The results are presented as a function of the oscillator spacing $\hbar \omega$ and the size of the model space $N$. As in the CCSD calculations, a Hartree-Fock basis has been used.

This speculation is borne out by two observations. First, the comparison of the UCOM interaction with the SRG interaction in Fig. 8 of Ref. [75] shows that the UCOM interaction does not decouple well in an oscillator basis. Second, the energy minima in Figs. 25, 26 and27 shift to higher frequencies $\hbar \omega$ as the size of the model space is increased, as one would expect based on the estimate (36) for an interaction with a large momentum cutoff. Our results also suggest that - similar to other SRG interactions and low-momentum interactions - the UCOM interaction has to be augmented by sizeable many-body forces [76]. The need for these forces is not seen if one restricts the calculations to fixed model spaces where model space parameters such as the oscillator frequency and the number of shells introduce additional momentum cutoffs.

\section{SUMMARY}

We presented a spherical formulation of the coupledcluster method for the computation of energy spectra in nuclei with closed subshells and their neighbors. This method has been used to solve nuclear structure problems in model spaces consisting of up to 20 oscillator shells, which allows us to obtain well-converged results for nucleon-nucleon interactions derived from chiral effective field theory. We find that chiral NN interactions saturate nuclei such as ${ }^{16} \mathrm{O}$ and ${ }^{48} \mathrm{Ca}$ within $0.5 \mathrm{MeV}$ per nucleon compared to data. The two-particle-two-hole clusters provide about $90 \%$ of correlation energy, with the approximation of triples clusters accounting for the remaining $10 \%$. We investigated the shell evolution in neutron-rich isotopes of oxygen and fluorine and found that nucleon-nucleon interactions alone fail to describe the experimentally observed (sub)shell structure. 
We also employed similarity renormalization group transformations of a "bare" chiral interaction. At the considered resolution scale, we obtained overbinding of up to several $\mathrm{MeV}$ per nucleon. Again, the missing contributions (when compared to experiment) are of natural size in the power counting of effective field theory. These results suggest that the systematic approach to nuclear structure can (in principle) be extended to medium-mass nuclei. We studied the $G$-matrix approach and found a very weak starting-energy dependence in large model spaces combined with a rather slow convergence with respect to increases in the size of the model space. For the UCOM interaction, we find a slow convergence with respect to the size of the model space and an overbinding for ${ }^{40} \mathrm{Ca}$ that is comparable to other low-momentum interactions.

We presented an in-depth study of the center-of-mass problem, and demonstrated that the wave functions of the intrinsic Hamiltonian factorize to a very good approximation into an intrinsic wave function and a Gaussian for the center of mass. While an analytical understanding of this behavior is still lacking, our calculations for ground and excited states and for a virtually exactly solvable toy model indicate that calculations in sufficiently large model spaces - when based on the intrinsic Hamiltonian - do not suffer from a center-of-mass problem.

\section{Appendix}

\section{COUPLED-CLUSTER DIAGRAMS IN A $J$-COUPLED SCHEME}

In this section we present expressions for several diagrams involved in our computation of the CCSD or $\Lambda$ $\operatorname{CCSD}(\mathrm{T})$ equations that involve various one-body, twobody, and three-body amplitudes and operators.

\section{A. Examples of CCSD diagrams in a $J$-coupled scheme}

We employ the following shorthands for the $T_{1}$ and $T_{2}$ amplitudes, namely $t_{i}^{a}$ and $t_{i j}^{a b}$, respectively. To the latter we add the total two-particle angular momentum $J_{i j}$ in order to indicate a two-body wave operator with hole states $i j$ coupled to a two-body angular momentum $J_{i j}$, that is

$$
\begin{gathered}
t_{i j}^{a b}\left(J_{i j}\right)=\left\langle\left(j_{a} j_{b}\right) J_{i j}|t|\left(j_{i} j_{j}\right) J_{i j}\right\rangle \\
=\sum_{m_{a} m_{b} m_{i} m_{j}} C_{m_{a} m_{b} M}^{j_{a} j_{b} J_{a b}} C_{m_{i} m_{i} M}^{j_{i j} j_{j} J_{i j}} \\
\left\langle\left(j_{a} m_{a}\right)\left(j_{b} m_{b}\right)|t|\left(j_{i} m_{i}\right)\left(j_{j} m_{j}\right)\right\rangle,
\end{gathered}
$$

with $m_{i}, m_{j}$, etc. being the magnetic quantum numbers of the corresponding single-particle angular momenta $j_{i}, j_{j}$, etc. The coefficients $C$ are the standard Clebsch-Gordan coefficients.
The two-body amplitudes are diagonal in the total angular momentum, that is, we have $J_{i j}=J_{a b}$. The final matrix elements are independent of $M$. The labels $a, b, c, d \ldots$ refer to particle states while $i, j, k, l, \ldots$ are hole states.

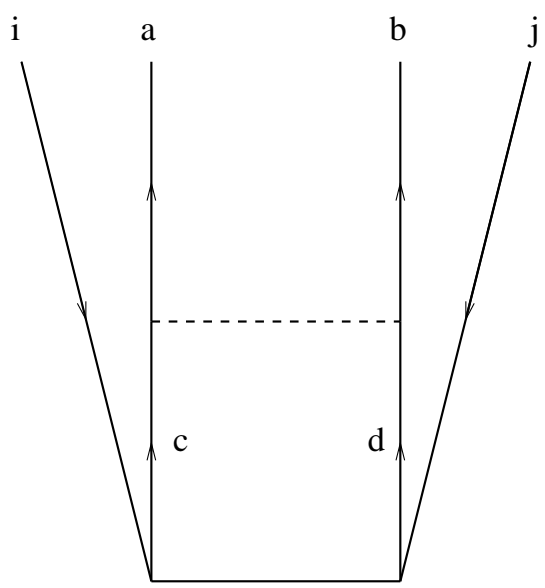

FIG. 28: (Color online) Diagram contributing to the $t_{i j}^{a b}(p p)$ amplitudes in particle-particle coupled scheme.

In Fig. 28 we give a diagram contributing to the $T_{2}$ equation, and the corresponding algebraic expression in the $J$-coupled scheme is (we list only the angular momentum related part),

$\tilde{t}_{i j}^{a b}\left(J_{i j}\right) \leftarrow \sum_{c d}\left\langle\left(j_{a} j_{b}\right) J_{a b}|V|\left(j_{c} j_{d}\right) J_{a b}\right\rangle\left\langle\left(j_{c} j_{d}\right) J_{a b}|t|\left(j_{i} j_{j}\right) J_{a b}\right\rangle$.

The above expression is obtained by identifying the diagram as a ladder cutting apart the lines connecting the various vertices. This technique is described in detail in Ref. [77]. Several applications of this technique are listed also in Ref. 222]. This is the most expensive diagram in the CCSD approximation and scales as $n_{o}^{2} n_{u}^{4}$, where $n_{o}$ is the number of occupied $j$-orbitals and $n_{u}$ is the number of unoccupied $j$-orbitals. As seen, the diagram can be calculated by a matrix-matrix multiplication utilizing efficient BLAS routines [78]. The diagram in Fig. 29]also

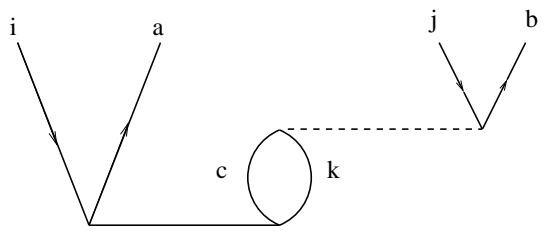

FIG. 29: (Color online) Diagram contributing to the $t_{i j}^{a b}(p h)$ amplitudes in particle-hole coupled scheme.

contributes to the $T_{2}$ equation, and is easiest calculated in a particle-hole coupled scheme so that intermediate sums of $9 j$-symbols are avoided. The transformation of the $t_{i j}^{a b}\left(J_{i j}\right)$ amplitudes to a particle-hole coupled scheme 
is given by

$$
\begin{aligned}
& \left\langle\left(j_{a} j_{i}^{-1}\right) J_{a i}|t|\left(j_{j} j_{b}^{-1}\right) J_{a i}\right\rangle= \\
& \quad \sum_{\substack{J_{a b}\\
}}\left(2 J_{a b}+1\right)(-1)^{j_{a}+j_{j}+J_{a b}+J_{a i}}\left\{\begin{array}{ccc}
j_{i} & j_{a} & J_{a i} \\
j_{b} & j_{j} & J_{a b}
\end{array}\right\} \times
\end{aligned}
$$

and the transformation back to particle-particle coupling is

$$
\begin{aligned}
& \left\langle\left(j_{a} j_{b}\right) J_{a b}|t|\left(j_{i} j_{j}\right) J_{a b}\right\rangle= \\
& \sum_{J_{a i}}\left(2 J_{a i}+1\right)(-1)^{j_{a}+j_{j}+J_{a b}+J_{a i}}\left\{\begin{array}{ccc}
j_{i} & j_{j} & J_{a b} \\
j_{b} & j_{a} & J_{a i}
\end{array}\right\} \times \\
& \left\langle\left(j_{a} j_{i}^{-1}\right) J_{a i}|t|\left(j_{j} j_{b}^{-1}\right) J_{a i}\right\rangle .
\end{aligned}
$$

The diagram in Fig. 29 can now easily be calculated in a particle-hole coupled scheme as,

$$
\begin{aligned}
& \left\langle\left(j_{a} j_{i}^{-1}\right) J_{a i}|\tilde{t}|\left(j_{j} j_{b}^{-1}\right) J_{a i}\right\rangle= \\
& \sum_{c k}(-1)^{j_{k}+j_{c}-J_{a i}+1}\left\langle\left(j_{a} j_{i}^{-1}\right) J_{a i}|t|\left(j_{k} j_{c}^{-1}\right) J_{a i}\right\rangle \times \\
& \left\langle\left(j_{k} j_{c}^{-1}\right) J_{a i}|V|\left(j_{j} j_{b}^{-1}\right) J_{a i}\right\rangle,
\end{aligned}
$$

where again an efficient BLAS [78] matrix-matrix multiplication routine can be used.

In Fig. 30 we give a diagram contributing to the $T_{1}$ equation. The algebraic expression of Fig. [30 in a $J$ -

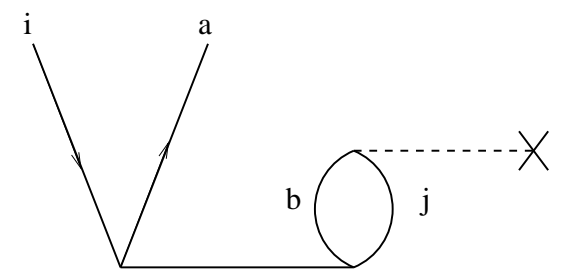

FIG. 30: (Color online) Diagram contributing to the $t_{i}^{a}$ amplitudes.

coupled scheme is,

$$
\tilde{t}_{i}^{a} \leftarrow \sum_{J_{i k}} \frac{2 J+1}{2 j_{a}+1} \sum_{c k} t_{a c}^{i k}\left(J_{i k}\right) \chi_{k}^{b},
$$

where $\chi_{k}^{b}$ is a one-body operator.

\section{B. Examples of $\Lambda$-CCSD(T) diagrams in a $J$-coupled scheme}

Figure 31 shows an example of diagrams with a threebody wave operator that give rise to contributions to the final energy. These diagrams arise from the so-called $\Lambda$-CCSD(T) approximation discussed in this work. The lower part of these diagrams is given by the three-body $t$ amplitude labeled $t_{i j k}^{a b c}$. It is antisymmetrized and in the

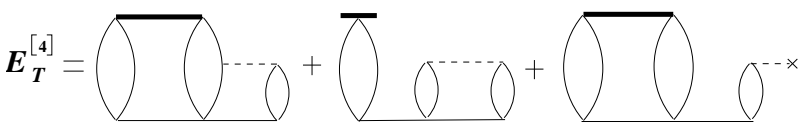

FIG. 31: (Color online) Diagrams for the $\Lambda$-CCSD(T) approximation.

$\Lambda$-CCSD $(\mathrm{T})$ approximation it is represented by a connected three-body contribution consisting of a two-body amplitude $t_{i j}^{a b}$ and a two-body interaction vertex plus a two-body amplitude part $t_{i j}^{a b}$ times a one-body amplitude $t_{k}^{c}$. These two contributions are depicted by the diagrams to the right of the equality sign in Fig. 32 . The threebody amplitude $t_{i j k}^{a b c}$ is shown to the left of the equality sign in Fig. 32 .

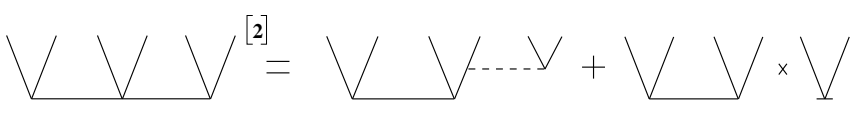

FIG. 32: (Color online) Diagrams for the $\Lambda$-CCSD(T) approximation.

A diagram like the first to the right of the equality sign in Fig. 32 can be redrawn as shown in Fig. 33 . Here we

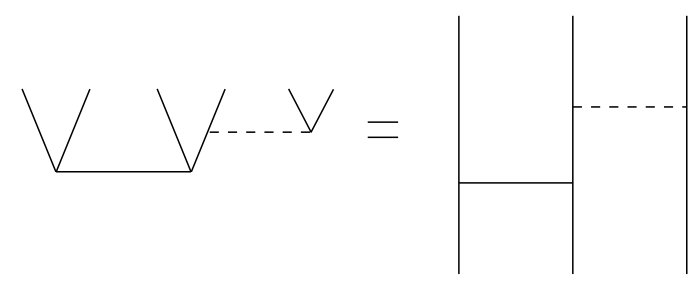

FIG. 33: (Color online) Connected part of three-particlethree-hole diagrams.

have stretched the hole lines in the diagram to the right of the equality sign. Such a diagram with, for example, an intermediate particle state can easily be calculated in an angular momentum coupled scheme. The expressions can be derived using the methods discussed in Ref. [77]. All three-body diagrams like those shown in Fig. 33 have been listed in Ref. 79].

In order to derive these expressions, one needs to specify the given coupling order for the angular momenta. Here we choose to couple our angular momenta as

$$
\begin{aligned}
& \left|\left(\left[j_{a} \rightarrow j_{b}\right] J_{a b} \rightarrow j_{c}\right) J\right\rangle= \\
& \quad \sum_{m_{a} m_{b} m_{c}}\left\langle j_{a} m_{a} j_{b} m_{b} \mid J_{a b} M_{a b}\right\rangle\left\langle J_{a b} M_{a b} j_{c} m_{c} \mid J M\right\rangle \\
& \left|j_{a} m_{a}\right\rangle \otimes\left|j_{b} m_{b}\right\rangle \otimes\left|j_{c} m_{c}\right\rangle,
\end{aligned}
$$

and indicated in Fig. 34

Note that the two-body intermediate state is antisymmetric but not normalized, that is, the state which involves the quantum numbers $j_{a}$ and $j_{b}$. We will hereafter 


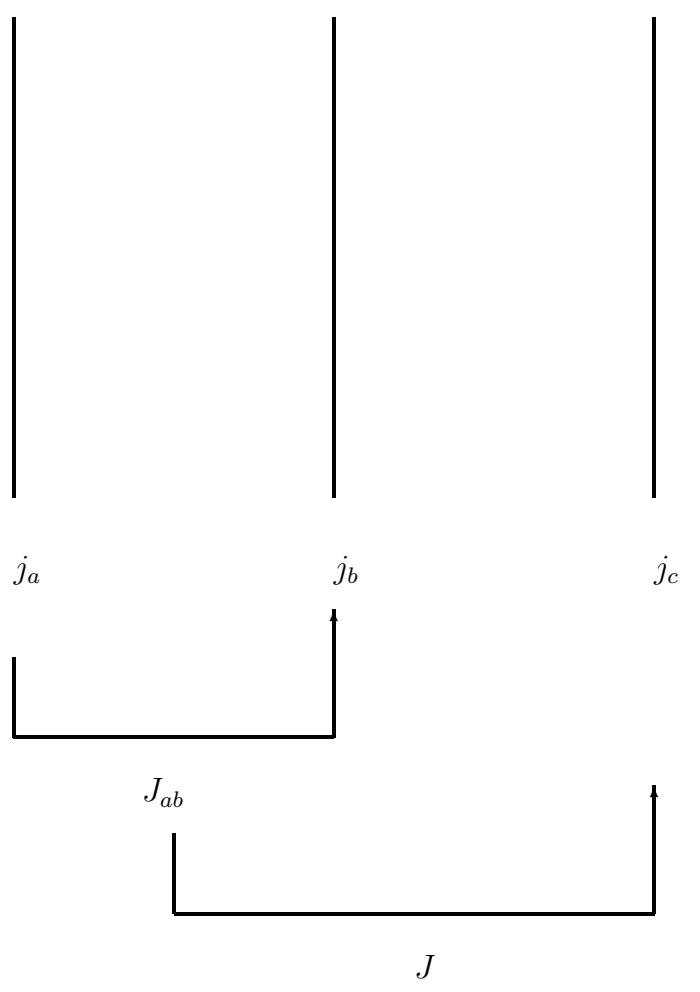

FIG. 34: The coupling order $\left(\left[j_{a} \rightarrow j_{b}\right] J_{a b} \rightarrow j_{c}\right) J$.

assume that the intermediate two-body state is antisymmetric. With this coupling order, we can rewrite the general three-particle Slater determinant as

$$
\Phi(1,2,3)=\mathcal{A}\left|\left(\left[j_{a} \rightarrow j_{b}\right] J_{a b} \rightarrow j_{c}\right) J\right\rangle
$$

with an implicit sum over $J_{a b}$. The final Slater determinant is

$$
\begin{aligned}
& \mathcal{A}\left|\left(\left[j_{a} \rightarrow j_{b}\right] J_{a b} \rightarrow j_{c}\right) J\right\rangle= \\
& \quad \frac{1}{\sqrt{3 !}}\left|\left(\left[j_{a} \rightarrow j_{b}\right] J_{a b} \rightarrow j_{c}\right) J\right\rangle- \\
& \frac{1}{\sqrt{3 !}}\left[\sum_{J_{a c}}(-1)^{j_{b}+j_{c}+J_{a b}+J_{a c}} \hat{J}_{a b} \hat{J}_{a c}\left\{\begin{array}{ccc}
j_{a} & j_{b} & J_{a b} \\
J & j_{c} & J_{a c}
\end{array}\right\}+\right. \\
& \left.\sum_{J_{b c}}(-1)^{J_{b c}} \hat{J}_{a b} \hat{J}_{b c}\left\{\begin{array}{ccc}
j_{a} & j_{b} & J_{a b} \\
J & j_{c} & J_{b c}
\end{array}\right\}\right]\left|\left(\left[j_{a} \rightarrow j_{b}\right] J_{a b} \rightarrow j_{c}\right) J\right\rangle .
\end{aligned}
$$

Here, we used the shorthand $\hat{J} \equiv \sqrt{2 J+1}$. With this coupling order, one can then compute all three-body diagrams like those listed in Fig. 33 .

Here we give an example of the closed form expression for the angular momentum recoupled part of a selected three-body diagram with two-body amplitudes $t_{i j}^{a b}$. These are diagrams which start with a three-body state but have two-body wave operators and end in a contribution to a three-body state with a two-body vertex after the two-body amplitude, as shown in Fig. 35 .
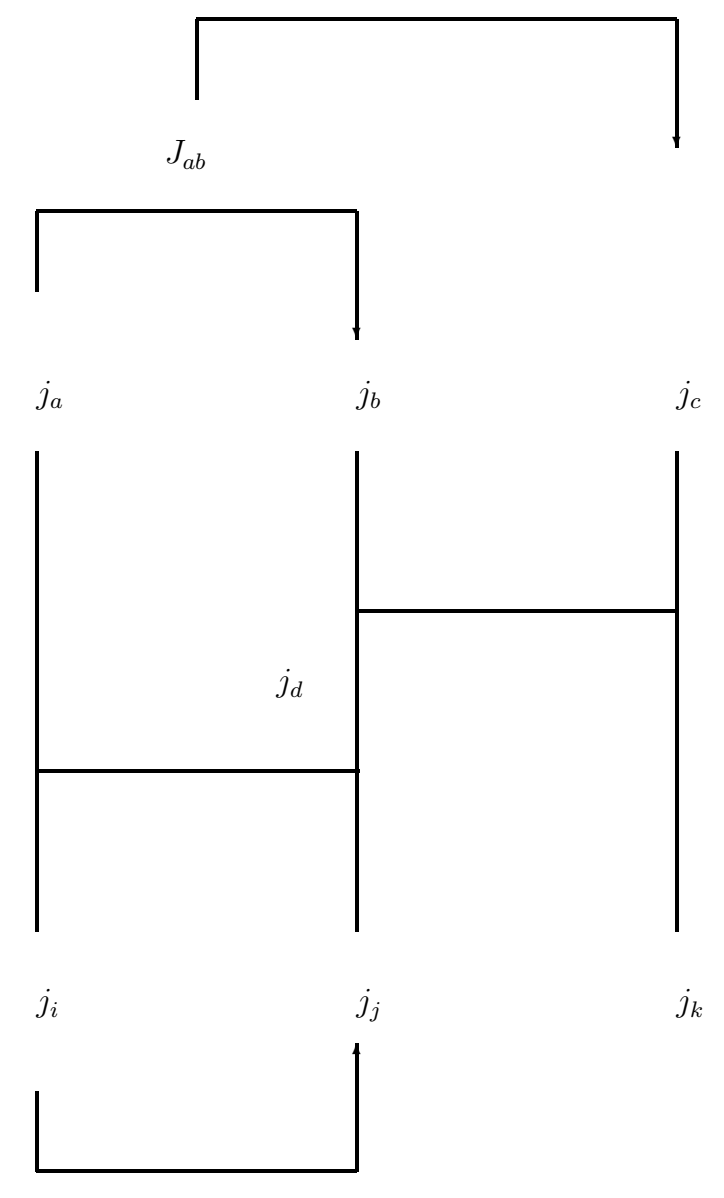

FIG. 35: Three-body diagram with two-body vertex and amplitude and a particle state as intermediate single-particle states. The sum over $d$ runs over all possible quantum numbers of the intermediate particle(hole) state. The algebraic expression of the above diagram, in an angular momentum coupled scheme, is given in Eq. (44).

Again, we employ the shorthand $t_{i j}^{a b}\left(J_{i j}\right)$ to indicate a two-body wave operator with hole states $i j$ coupled to a two-body angular momentum $J_{i j}$, that is, $t_{a b}^{i j}\left(J_{i j}\right)=$ $\left\langle\left(j_{a} j_{b}\right) J_{i j}|t|\left(j_{i} j_{j}\right) J_{i j}\right\rangle$. The diagram with a particle intermediate state is shown in Fig. 35] with its angular momentum representation,

$$
\begin{gathered}
\sum_{J_{b c}} \sum_{d}(-1)^{j_{b}+j_{c}+j_{d}+J_{b c}} \hat{J}_{a b} \hat{J}_{i j} \hat{J}_{b c}^{2}\left\{\begin{array}{ccc}
j_{a} & j_{b} & J_{a b} \\
j_{c} & J & J_{b c}
\end{array}\right\} \\
\left\{\begin{array}{ccc}
j_{a} & j_{d} & J_{i j} \\
j_{k} & J & J_{b c}
\end{array}\right\}\left\langle\left(j_{b} j_{c}\right) J_{b c}|V|\left(j_{d} j_{k}\right) J_{b c}\right\rangle t_{a d}^{i j}\left(J_{i j}\right) .
\end{gathered}
$$

The expression for this diagram is obtained by opening up the intermediate three-particle state $j_{a} j_{d} j_{k}$ and recoupling the angular momenta $j_{b}$ and $j_{c}$ to yield a final two-particle angular momentum $J_{b c}$. This applies also to the single-particle angular momenta $j_{d}$ and $j_{k}$. These two single-particle angular momenta couple to the same final 
two-particle angular momentum $J_{b c}$. These recouplings are reflected in the two $6 j$-symbols in the above expression. The expression for the corresponding diagram of Fig. 35 with a hole intermediate state is obtained by replacing the particle labeling $j_{d}$ with $j_{l}$. The subscript $l$ refers to the fact that this is a single-hole state. The internal hole line, however, gives rise to a factor -1 .

In total there are, due to the antisymmetry of the three-body wave function, nine diagrams with an intermediate particle state and nine diagrams with an intermediate single-hole state. The angular momentum expressions for these diagrams are listed in Ref. [79]. The expressions for diagrams like the rightmost one in Fig. 32 yield similar expressions and are easy to compute. They represent a two-body part coupled with a one-body part.

We thank S.K. Bogner, R.J. Furnstahl, and A. Schwenk for useful discussions. This work was supported by the Office of Nuclear Physics, U.S. Department of Energy (Oak Ridge National Laboratory); the University of Washington under Contract No. DE-FC02-07ER41457; and the University of Tennessee under Grant No. DEFG02-96ER40963. MHJ thanks the Research Council of Norway for support.
[1] S. C. Pieper and R. B. Wiringa, Ann. Rev. Nucl. Part. Sci. 51, 53 (2001).

[2] P. Navrátil, J.P. Vary, and B.R. Barrett, Phys. Rev. C 62, 054311 (2000).

[3] A. Nogga, in Proceedings of Chiral Dynamics 2006, Eds. Mohammad W. Ahmed, Haiyan Gao, Henry R. Weller, and Barry Holstein, (World Scientific, Singapore, 2007); arXiv:nucl-th/0611081.

[4] A. Kievsky, S. Rosati, M. Viviani, L. E. Marcucci, and L. Girlanda, J. Phys. G 35, 063101 (2008).

[5] E. Epelbaum, H. Krebs, D. Lee, and U.-G. Meißner Phys. Rev. Lett.104, 142501 (2010).

[6] P. Navrátil, V. G. Gueorguiev, J. P. Vary, W.E. Ormand, and A. Nogga, Phys. Rev. Lett. 99, 042501 (2007).

[7] Petr Navrátil, Sofia Quaglioni, Ionel Stetcu, and Bruce R. Barrett, J. Phys. G 36, 08310 (2009).

[8] G. Hagen, T. Papenbrock, D. J. Dean, and M. HjorthJensen, Phys. Rev. Lett. 101, 092502 (2008).

[9] S. Bacca, N. Barnea, W. Leidemann, and G. Orlandini, Phys. Rev. Lett.102, 162501 (2009).

[10] S. Weinberg, Phys. Lett. B 251, 288 (1990); Nucl. Phys. B 363, 3 (1991).

[11] U. van Kolck, Phys. Rev. C 49, 2932 (1994).

[12] E. Epelbaum, W. Glöckle, and U. G. Meißner, Nucl. Phys. A 671, 295 (2000).

[13] D. R. Entem and R. Machleidt, Phys. Rev. C 68, 041001(R) (2003).

[14] E. Epelbaum, W. Glöckle, and U. G. Meißner, Nucl. Phys. A 747, 362 (2005).

[15] D. R. Entem and R. Machleidt, Phys. Lett. B524, 93 (2002).

[16] N. Michel et al, arXiv:1002.0770 (2010).

[17] G. Hagen, D.J. Dean, M. Hjorth-Jensen, and T. Papenbrock, Phys. Lett. B 656, 169 (2007).

[18] G. Hagen, T. Papenbrock, and M. Hjorth-Jensen, Phys. Rev. Lett. 104, 182501 (2010).

[19] S. K. Bogner, R. J. Furnstahl and R. J. Perry, Phys. Rev. C 75, 061001 (2007).

[20] S. K. Bogner, T. T. S. Kuo, A. Schwenk, D. R. Entem, and R. Machleidt, Phys. Lett. B 576, 265 (2003).

[21] S.K. Bogner, T.T.S. Kuo, and A. Schwenk, Phys. Rept. 386, 1 (2003).

[22] M. Hjorth-Jensen, T. T. S. Kuo, and E. Osnes, Phys. Rep. 261, 125 (1995).

[23] W. H. Dickhoff and D. Van Neck, Many-body Theory exposed!: Propagator Description of Quantum Mechan- ics in Many-body Systems, (World Scientific, Hackensack, NJ, 2005).

[24] H. Feldmeier, T. Neff, R. Roth, and J. Schnack, Nucl. Phys. A 632, 61 (1998).

[25] D. J. Dean, G. Hagen, M. Hjorth-Jensen, T. Papenbrock, and A. Schwenk, Phys. Rev. Lett. 101, 119201 (2008).

[26] G. Hagen, T. Papenbrock, D. J. Dean, Phys. Rev. Lett. 103, 062503 (2009).

[27] G. Hagen, T. Papenbrock, D. J. Dean, M. Hjorth-Jensen, and B. Velamur Asokan, Phys. Rev. C 80, 021306(R) (2009).

[28] F. Coester, Nucl. Phys. 7, 421 (1958).

[29] F. Coester and H. Kümmel, Nucl. Phys. 17, 477 (1960).

[30] J. Čížek, J. Chem. Phys. 45, 4256 (1966).

[31] J. Č́žzek, Adv. Chem. Phys. 14, 35 (1969).

[32] H. Kümmel, K.H. Lührmann, and J.G. Zabolitzky, Phys. Rep. 36, 1 (1978).

[33] R.J. Bartlett and M. Musiał, Rev. Mod. Phys. 79, 291 (2007)

[34] R. F. Bishop, M. F. Flynn, M. C. Boscá, E. Buendá, and R. Guardiola, Phys. Rev. C 42, 1341 (1990).

[35] J.H. Heisenberg and B. Mihaila, Phys. Rev. C 59, 1440 (1999).

[36] B. Mihaila and J.H. Heisenberg, Phys. Rev. C 61, 054309 (2000); Phys. Rev. Lett. 84, 1403 (2000).

[37] D.J. Dean and M. Hjorth-Jensen, Phys. Rev. C 69, 054320 (2004).

[38] K. Kowalski, D.J. Dean, M. Hjorth-Jensen, T. Papenbrock, and P. Piecuch, Phys. Rev. Lett. 92, 132501 (2004).

[39] M. Włoch, D.J. Dean, J.R. Gour, M. Hjorth-Jensen, K. Kowalski, T. Papenbrock, and P. Piecuch, Phys. Rev. Lett. 94, 212501 (2005).

[40] Y.S. Lee, S.A. Kucharski, R.J. Bartlett, J. Chem. Phys. 81, 5906 (1984); ibid 82, 761 (E) (1982). J. Noga, R.J. Bartlett, M. Urban, Chem. Phys. Lett. 134, 126 (1987).

[41] M. J. O. Deegan and P. J. Knowles, Chem. Phys. Lett. 227, 321 (1994).

[42] A. D. Taube and R. J. Bartlett, J. Chem. Phys. 128, 044110 (2008).

[43] S. A. Kucharski and R. J. Bartlett, J. Chem. Phys. 108, 5243 (1998).

[44] R. J. Bartlett, Int. J. Mol. Sci. 3, 579 (2002).

[45] D. J. Rowe, Rev. Mod. Phys., 40, 153 (1968).

[46] H. Sekino and R. J. Bartlett, Int. J. Quantum Chem., Quantum Chem. Symp. 18 , 255 (1984). 
[47] S. Hirata, M. Nooijen, R. J. Bartlett, Chem. Phys. Lett. 328, 459 (2000).

[48] R. Roth, J. R. Gour, P. Piecuch, Phys. Lett. B 679, 334 (2009).

[49] D. H. Gloeckner and R. D. Lawson, Phys. Lett. B 53, 313 (1974).

[50] J. B. McGrory and B. H. Wildenthal, Phys. Lett. B 60, 5 (1975).

[51] S. Quaglioni and P. Navrátil, Phys. Lett. B 652, 370 (2007).

[52] S. Fujii, R. Okamoto, K. Suzuki, Phys. Rev. Lett. 103, 182501 (2009).

[53] S. Fujii, R. Okamoto, and K. Suzuki, J. Phys. Conf. Ser. 20, 83 (2005).

[54] O. Sorlin and M. G. Porquet, Prog. Part. Nucl. Phys. 61, 602 (2008).

[55] P. G. Thirolf et al, Phys. Lett. B485, 16 (2000).

[56] R. Kanungo et al., Phys. Rev. Lett. 102, 152501 (2009).

[57] M. Stanoiu et al, Phys. Rev. C 69, 034312 (2004).

[58] C. R. Hoffman et al, Phys. Lett. B672, 17 (2009).

[59] Y. Luo, J. Okolowicz, M. Ploszajczak, and N. Michel, nucl-th/0211068 (2002).

[60] N. Michel, W. Nazarewicz, M. Ploszajczak, and J. Okolowicz, Phys. Rev. C 67054311 (2003).

[61] A. Volya and V. Zelevinsky, Phys. Rev. C 74, 064314(2006).

[62] T. Otsuka, T. Suzuki, J. D. Holt, A. Schwenk, and Y. Akaishi, arXiv:0908.2607 (2009).

[63] K. Tsukiyama, M. Hjorth-Jensen, G. Hagen, Phys. Rev. C 80, 051301(R) (2009).

[64] A. Zuker, Phys. Rev. Lett. 90, 042502 (2003).

[65] E. Epelbaum, A. Nogga, W. Glöckle, H. Kamada, Ulf-G.
Meißner, and H. Witala, Phys. Rev. C66, 064001 (2002).

[66] A. Nogga, S. K. Bogner, and A. Schwenk, Phys. Rev. C 70, 061002 (2004).

[67] E. D. Jurgenson, P. Navrátil, and R. J. Furnstahl, Phys. Rev. Lett. 103, 082501 (2009).

[68] N. Michel, W. Nazarewicz, and M. Ploszajczak, Phys. Rev. C 75, 031301 (2007).

[69] Sofia Quaglioni and Petr Navrátil, Phys. Rev. Lett. 101, 092501 (2008).

[70] G. Hagen, D. J. Dean, M. Hjorth-Jensen, T. Papenbrock and A. Schwenk, Phys. Rev. C 76, 044305 (2007).

[71] R. Roth, P. Papakonstantinou, N. Paar, H. Hergert, T. Neff, H. Feldmeier, Phys.Rev. C 73, 044312 (2006).

[72] R. Roth, J.R. Gour, and P. Piecuch, Phys. Rev. C 79, 054325 (2009).

[73] R. Roth and P. Navrátil, Phys. Rev. Lett. 99, 092501 (2007).

[74] Robert Roth, Thomas Neff, and Hans Feldmeier, arXiv:1003.3624 (2010).

[75] R. Roth, S. Reinhardt, and H. Hergert, Phys. Rev. C 77, 064003 (2008).

[76] M. Hjorth-Jensen, G. Hagen, D. J. Dean, and S. Kvaal, J. Phys. G 37, 064035 (2010).

[77] T. T. S. Kuo, J. Shurpin, K. C. Tam, E. Osnes, and P. J. Ellis, Ann. Phys. 132, 237 (1981).

[78] For a full documentation of the B(asic)L(inear)A(lgebra)S(ubprograms) software, see http://www .netlib.org/blas.

[79] A. Polls, H. Müther, A. Faessler, T. T. S. Kuo, and E. Osnes, Nucl. Phys. A401, 124 (1983). 